\title{
A NOTION OF RANK FOR UNITARY REPRESENTATIONS OF REDUCTIVE GROUPS BASED ON KIRILLOV'S ORBIT METHOD
}

\author{
HADI SALMASIAN
}

\begin{abstract}
We introduce a new notion of rank for unitary representations of semisimple groups over a local field of characteristic zero. The theory is based on Kirillov's method of orbits for nilpotent groups over local fields. When the semisimple group is a classical group, we prove that the new theory is essentially equivalent to Howe's theory of $N$-rank [Hw1, Li1], Sca. Therefore, our results provide a systematic generalization of the notion of a small representation (in the sense of Howe) to exceptional groups. However, unlike previous works which used ad-hoc methods to study different types of classical groups (and some exceptional ones $\underline{\underline{\mathrm{WS}}}, \underline{\mathrm{LS}}$ ), our definition is simultaneously applicable to both classical and exceptional groups. The most important result of this paper is a general "purity" result for unitary representations, which demonstrates how similar partial results in the previous authors' works should be formulated and proved for an arbitrary semisimple group in the language of Kirillov's theory. The purity result is a crucial step towards studying small representations of exceptional groups. New results concerning small unitary representations of exceptional groups will be published in a forthcoming paper $\mathrm{Sa}$.
\end{abstract}

\section{CONTEnts}

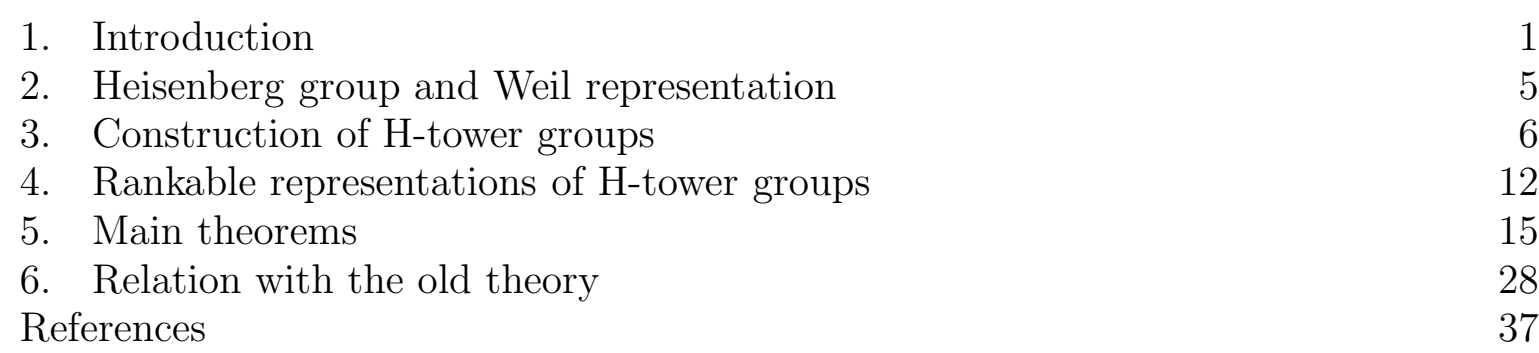

\section{INTRODUCTION}

1.1. Background. The theory of $N$-rank [Hw1, [Li1], Sca provides an effective tool for studying "small" unitary representations of classical semisimple groups. It is based on analysing the restriction of a unitary representation to large commutative subgroups of such groups. The study of the notion of $N$-rank began in [Hw1] where the theory was developed for the metaplectic group. Roughly speaking, one can attach equivalence classes of bilinear symmetric forms to unitary representations, and these equivalence classes canonically correspond to orbits of the adjoint action of the Levi component of

Date: August 282005.

2000 Mathematics Subject Classification. 22E46,22E50,11F27.

Key words and phrases. Unitary representations, Kirillov's method of orbits. 
the Siegel parabolic on its unipotent radical. Later on, the theory was extended by J. S. Li [Li1] and R.Scaramuzzi [Sca] to classical groups, and an intrinsic connection with the theta correspondence was also found in [Li2]. Representations which correspond to degenerate classes are called singular representations. They form a large class of nontempered representations and therefore their study is essentially complementary to that of the tempered representations. It turns out that this class of representations is quite well-behaved. In fact Howe gave a construction of a large class of such representations [Hw3], and they are applied in construction of automorphic forms [Hw4], [Li3].

The lack of a theory of $N$-rank which applies to the exceptional groups is in a sense a defect of this theory due to substantial interest in the small representations of these groups. Aside from some generalizations in the works of Loke and Savin [LS] and Sahi, most recently Weissman Ws provides an analogue for $p$-adic split simply-laced groups excluding $E_{8}$ using the representation-theoretic analogue of a Fourier-Jacobi functor.

Here we develop a theory of rank which can also be applied to (almost) all exceptional groups. The word "almost" will be explained more precisely in the remark after Proposition 3.1.1. Our theory relies on the structure of unipotent radicals of certain parabolic subgroups which are naturally expressible as a sequence of extensions by Heisenberg groups. In this paper these unipotent radicals are called $H$-tower groups (see Definition 3.2.5).

The main point of this work is to show that although the abelian nilradicals in terms of which rank was defined for classical groups are usually not available in exceptional cases, the maximal unipotent subgroups of all semisimple groups have a common feature which enables us to define a similar notion of rank, which is essentially equivalent to the older one for classical groups. This result, apart from being interesting itself, provides the main tool needed to study small representations of exceptional groups. One interesting application of this result is that it enables us to obtain sharp bounds on the behaviour of matrix coefficients of unitary representations of exceptional groups, a problem studied in [LZ, [Oh] and [LS]. Results along this line of research will be published elsewhere [Sa].

It is also possible to generalize Theorem 4.2 of [Hw1, which is the next step towards classification of small representations of exceptional groups. However, there does not seem to be any strong connection between the "exceptional theta correspondences" which result from the author's work and the ones that already exist in the literature (see [DS, MS or [GRS for instance). This makes the classification problem both harder and more interesting at the same time. This is a work still in its preliminary stages.

This manuscript is organized as follows. Section [1.2 is devoted to the notation used throughout the article. In section 2 we recall some basics about the Heisenberg groups and the Weil representation. In section 3 we define a specific unipotent subgroup of a simple group with regard to which our rank is defined. The "rankable" representations of this unipotent group are defined and studied briefly in section 4 . Section 5 is devoted to the statement and proof of the main theorems, which essentially assert that rank is a nontrivial invariant of a representation. Section 6 shows why our new theory generalizes the older one. 
Acknowledgement. This paper is part of the author's thesis written under Roger Howe's supervision. The author thanks him for his support and encouragement. The author thanks Igor Frenkel, Gopal Prasad, Gordan Savin, Siddhartha Sahi, Jeb Willenbring and Gregg Zuckerman for fruitful conversations/communications, and especially the anonymous referee for providing the author with several extremely helpful suggestions and for mentioning the inaccuracies which existed in section 5 of an earlier version of the paper.

1.2. Notation. In this section we introduce some notation which will be used throughout this article. Throughout this paper, we will be working with a local field $\mathbb{F}$ which is either $\mathbb{R}, \mathbb{C}$ or a finite extension of $\mathbb{Q}_{p}, p \neq 2$. Let $\overline{\mathbb{F}}$ be the algebraic closure of $\mathbb{F}$, and $\mathbf{G}$ be the $\overline{\mathbb{F}}$-points of an absolutely simple, simply connected linear algebraic group defined over $\mathbb{F}$, and $\mathbf{G}_{\mathbb{F}}$ be the group of $\mathbb{F}$-rational points of $\mathbf{G}$. We assume $\mathbf{G}$ is $\mathbb{F}$-isotropic. By the Kneser-Tits conjecture, which holds in the case of local fields (see Proposition 7.6 and Theorem 7.6 in $[\mathrm{PR}, \S 7.2]), \mathbf{G}_{\mathbb{F}}$ is equal to the group generated by its unipotent elements. (In the archimedean case, $\mathbf{G}_{\mathbb{F}}$ is a connected Lie group.) Let $G$ be a finite topological central extension of $\mathbf{G}_{\mathbb{F}}$. (For the definition of a topological central extension, the reader is referred to Mo2, Chapter 1].)

Take a maximally split Cartan subgroup $\mathbf{H}$ of $\mathbf{G}$ (which is also defined over $\mathbb{F}$ ), and let $\mathbf{A}$ be the maximal split torus inside $\mathbf{H}$. Let $A$ be the inverse image of $\mathbf{A}_{\mathbb{F}}$ in $G$.

We denote the Lie algebras of the groups $\mathbf{G}, \mathbf{H}, \mathbf{A}, G, A$ by $\mathfrak{g}, \mathfrak{h}, \mathfrak{a}, \mathfrak{g}_{\mathbb{F}}, \mathfrak{a}_{\mathbb{F}}$ respectively. More precisely, $\mathfrak{g}, \mathfrak{h}, \mathfrak{a}$ are $\overline{\mathbb{F}}$-Lie algebras and $\mathfrak{g}_{\mathbb{F}}$ and $\mathfrak{a}_{\mathbb{F}}$ are $\mathbb{F}$-forms of the corresponding algebras.

Let $\Delta$ be an absolute root system associated to $\mathfrak{h}$, and let $\Sigma$ be the restricted (or relative, as some people call it) root system associated to $\mathfrak{a}$. The root space corresponding to any $\alpha \in \Delta$ is denoted by $\mathfrak{g}_{\alpha}$, and the coroot for $\alpha$ is denoted by $H_{\alpha}$.

We fix a G-invariant symmetric bilinear form $(\cdot, \cdot)$ on $\mathfrak{g}$, normalized in such a way that if for any two roots $\beta, \gamma$ we define $(\beta, \gamma)$ to be $\left(H_{\beta}, H_{\gamma}\right)$, then $(\beta, \beta)=2$ for any long root $\beta$. All roots of simply-laced root systems are considered as long ones. For $\mathfrak{h}_{\mathbb{F}}=\mathfrak{h} \cap \mathfrak{g}_{\mathbb{F}}$ we have

$$
\mathfrak{h}_{\mathbb{F}}=\mathfrak{a}_{\mathbb{F}} \oplus \mathfrak{t}_{\mathbb{F}}
$$

where $\mathfrak{t}_{\mathbb{F}}$ is the orthogonal complement of $\mathfrak{a}_{\mathbb{F}}$ in $\mathfrak{h}_{\mathbb{F}}$ with respect to the invariant symmetric bilinear form $(\cdot, \cdot)$ introduced above.

As usual, $\Delta^{+}$and $\Sigma^{+}$denote the set of positive roots. We assume $\Delta^{+}$, when restricted to $\mathfrak{a}$, contains $\Sigma^{+}$. Let $\Delta_{B}$ and $\Sigma_{B}$ denote the bases for positive roots in the corresponding root systems such that $\Delta_{B}$, when restricted to $\mathfrak{a}$, contains $\Sigma_{B}$.

The choice of a positive system determines a fixed Borel subalgebra $\mathfrak{b}$ of $\mathfrak{g}$ (or a minimal parabolic $\mathfrak{p}_{\text {min }}$ of $\left.\mathfrak{g}_{\mathbb{F}}\right)$. A parabolic subalgebra of $\mathfrak{g}$ (respectively, of $\mathfrak{g}_{\mathbb{F}}$ ) is called standard if it contains $\mathfrak{b}$ (respectively, $\mathfrak{p}_{\min }$ ). We identify the standard parabolic subgroups and subalgebras of $\mathfrak{g}$ (respectively of $\mathfrak{g}_{\mathbb{F}}$ ) by subsets of $\Delta_{B}$ (respectively of $\Sigma_{B}$ ) they naturally correspond to. More precisely, for any subset $\Phi \subseteq \Delta_{B}$, by $\mathfrak{p}_{\Phi}$ we mean the standard 
parabolic subalgebra of $\mathfrak{g}$ which contains all root spaces $\mathfrak{g}_{\alpha}$ for any $\alpha \in \Delta^{+}$as well as any $\mathfrak{g}_{-\alpha^{\prime}}$ such that $\alpha^{\prime} \in \Delta^{+}$is in the semigroup generated by $\Phi$. The definition will be similar for the parabolic subalgebra (this time of $\mathfrak{g}_{\mathbb{F}}$ ) corresponding to any $\Phi \subseteq \Sigma_{B}$. Later on, when there is no risk of confusion between the parabolics of $\mathfrak{g}$ and $\mathfrak{g}_{\mathbb{F}}$, we use the more concise notation $\mathfrak{p}_{\Phi}$ for both situations. Also, again for simplicity and when there is no risk of ambiguity, Lie subalgebras of both $\mathfrak{g}$ and $\mathfrak{g}_{\mathbb{F}}$ may be denoted by Gothic letters in a similar way; i.e. without a subscript " $\mathbb{F}$ " in the case of a Lie subalgebra of $\mathfrak{g}_{\mathbb{F}}$.

The standard parabolic subgroups of $\mathbf{G}$ and $G$ are denoted by $\mathbf{P}_{\Phi}$ and $P_{\Phi}$ respectively. Note that a parabolic subgroup of $G$ means the inverse image of a parabolic subgroup of $\mathbf{G}_{\mathbb{F}}$ in $G$. In other words, if the short exact sequence

$$
1 \longrightarrow F \stackrel{i}{\longrightarrow} G \stackrel{p}{\longrightarrow} \mathbf{G}_{\mathbb{F}} \longrightarrow 1
$$

(where $F$ is a finite subgroup of the center of $G$ ) represents our topological central extension, then a parabolic $P$ of $G$ is of the form $P=p^{-1}\left(\mathbf{P}_{\mathbb{F}}\right)$ for an $\mathbb{F}$-parabolic $\mathbf{P}$ of G. It follows from [Du, II 11 Lemme] (or from an explicit construction of the universal central extension by Deodhar [De, Section 1.9]) that topological central extensions of semisimple groups over local fields are split over the unipotent subgroups ${ }^{1}$ and therefore one can have an analogous "Levi" decomposition in $G$ as well; i.e. if $P=p^{-1}\left(\mathbf{P}_{\mathbb{F}}\right)$ and $\mathbf{P}=\mathbf{L} \mathbf{N}$ is a Levi decomposition of $\mathbf{P}$ (with $\mathbf{L}$ being the reductive factor and $\mathbf{N}$ being the unipotent radical) then one can express $P$ as $P=L N$, where:

1. $L=p^{-1}\left(\mathbf{L}_{\mathbb{F}}\right)$.

2. $N \subseteq p^{-1}\left(\mathbf{N}_{\mathbb{F}}\right)$ is a normal subgroup of $P$ and the map

$$
p: N \mapsto \mathbf{N}_{\mathbb{F}}
$$

is an isomorphism of topological groups. (Existence of $N$ is what follows from the results of Duflo or Deodhar cited above.)

We may as well drop the subscript $\Phi$ of the standard parabolics $P_{\Phi}$ and $\mathbf{P}_{\Phi}$ when there is no risk of confusion about the identifying subset $\Phi$ of simple positive roots. Moreover, whenever a parabolic subgroup or subalgebra is standard, we assume that the Levi decomposition considered above is also standard.

Let $K$ be an arbitrary locally compact group. Then the center of $K$ will be denoted by $\mathcal{Z}(K)$. Unitary representations of $K$ are defined in the usual way (see [Mac1]) and denoted by Greek letters. The unitary dual of $K$ (which is the set of equivalence classes of the irreducible unitary representations of $K$ endowed with the Fell topology) is denoted by $\hat{K}$. Now suppose $K^{\prime}$ is a closed subgroup of $K$ and let $\sigma, \sigma^{\prime}$ be unitary representations of $K, K^{\prime}$ respectively. Then, as usual, $\operatorname{Res}_{K^{\prime}}^{K} \sigma$ and $\operatorname{Ind}_{K^{\prime}}^{K} \sigma^{\prime}$ mean restriction and unitary induction. If there is no ambiguity about $K$, we use the simpler notation $\sigma_{\mid K^{\prime}}$ instead of $\operatorname{Res}_{K^{\prime}}^{K} \sigma$.

Suppose $\mathfrak{l}$ is an arbitrary Lie algebra. Then the center of $\mathfrak{l}$ is denoted by $\mathfrak{z}(\mathfrak{l})$.

\footnotetext{
${ }^{1}$ In fact an abstract (and hence topological) universal central extension of $\mathbf{G}_{\mathbb{F}}$ splits over the maximal unipotent subgroup. I am indebted to Gopal Prasad for pointing to reference De.
} 


\section{Heisenberg group AND Weil REPRESEntation}

2.1. Heisenberg groups and algebras. Let $n$ be a positive integer. An $\mathbb{F}$-Heisenberg group (or simply a Heisenberg group) $H_{n}$ is a two-step nilpotent $2 n+1$-dimensional $\mathbb{F}$-group which is isomorphic to the subgroup of $G L_{n+2}(\mathbb{F})$ of unipotent, upper-triangular matrices which do not have nonzero elements outside the diagonal, the first row and the last column. This group has a one-dimensional center. We denote the Lie algebra of $H_{n}$ by $\mathfrak{h}_{n}$. Since $H_{n} \subset G L_{n+2}(\mathbb{F}), \mathfrak{h}_{n}$ sits in the usual way inside $\mathfrak{g l}_{n+2}(\mathbb{F})$.

The Lie algebra $\mathfrak{h}_{n}$ of $H_{n}$ can also be described more abstractly as

$$
\mathfrak{h}_{n}=W_{n} \oplus \mathfrak{z}
$$

with the Lie bracket introduced below, where $W_{n}$ is a $2 n$-dimensional space endowed with a nondegenerate symplectic form $\langle\cdot, \cdot\rangle$, and $\mathfrak{z}$ is the center of $\mathfrak{h}_{n}$, a one-dimensional Lie subalgebra. We fix a Lie algebra isomorphism between $\mathfrak{z}$ and $\mathbb{F}$, and denote the element in $\mathfrak{z}$ corresponding to $1 \in \mathbb{F}$ by $Z$. The Lie bracket on $W_{n}$ is defined as follows:

$$
\text { for every } X, Y \in W_{n}, \quad[X, Y]=<X, Y>Z \text {. }
$$

Viewing $\mathfrak{h}_{n}$ as a subalgebra of $\mathfrak{g l}_{n+2}(\mathbb{F})$, the exponential map of $G L_{n+2}(\mathbb{F})$ gives a bijection between $\mathfrak{h}_{n}$ and $H_{n}$, and $\mathcal{Z}\left(H_{n}\right)$ is equal to $\exp (\mathfrak{z})$. This bijection is the exponential map of $H_{n}$.

2.2. Stone-von Neumann theorem. We briefly mention the structure of irreducible, infinite-dimensional unitary representations of a Heisenberg group. For a more detailed discussion, the reader can study [Hw2], CG] or Tay, Chapter 1] for instance.

The Stone-von Neumann theorem states that for any nontrivial unitary character of $\mathcal{Z}\left(H_{n}\right)$, up to unitary equivalence there is a unique infinite-dimensional irreducible unitary representation of $H_{n}$ having this central character. We will describe an explicit realization (the so-called Schrödinger model) for this representation below.

Consider an arbitrary polarization of $W_{n}$, i.e. a decomposition of $W_{n}$ as a direct sum

$$
W_{n}=\mathfrak{x}_{n} \oplus \mathfrak{y}_{n}
$$

of maximal isotropic subspaces. It is possible to choose bases $\left\{X_{1}, \ldots, X_{n}\right\}$ and $\left\{Y_{1}, \ldots, Y_{n}\right\}$ of $\mathfrak{x}_{n}$ and $\mathfrak{y}_{n}$ respectively such that

$$
<X_{i}, Y_{j}>=\delta_{i, j}
$$

There is an isomorphim

$$
e: \mathfrak{x}_{n} \approx \mathfrak{y}_{n}^{*}
$$

obtained via the symplectic form. $\mathcal{Z}\left(H_{n}\right) \approx \exp (\mathbb{F})$ and any nontrivial multiplicative (unitary) character of $\mathcal{Z}\left(H_{n}\right)$ is equal to $\chi \circ \exp ^{-1}$, for some $\chi$, where $\chi$ can be any nontrivial additive (unitary) character of $\mathfrak{z}\left(\mathfrak{h}_{n}\right) \approx \mathbb{F}$. Such characters $\chi$ are in one-to-one correspondence with elements of $\mathbb{F}-\{0\}$. For example, if $\mathbb{F}=\mathbb{R}, \chi$ will be of the form $\chi(x)=e^{\mathrm{i} x y}$ for some $y \in \mathbb{R}-\{0\}$ (here $\mathrm{i}=\sqrt{-1}$ ). This follows from the well-known fact that the unitary dual of a local field is identifiable to itself (see We1]). 
The (irreducible) representation $\rho$ of $H_{n}$ with central character $\chi$ may be realized on the Hilbert space

$$
\mathcal{H}_{\rho}=L^{2}\left(\mathfrak{y}_{n}\right)
$$

as follows. Let $y=\sum_{i=1}^{n} y_{i} Y_{i} \in \mathfrak{y}_{n}$. Then $H_{n}$ acts on $\mathcal{H}_{\rho}$ as

$$
\begin{aligned}
\left(\rho\left(e^{t X_{i}}\right) f\right)(y) & =\chi\left(-t y_{i}\right) f(y) \\
\left(\rho\left(e^{t Y_{i}}\right) f\right)(y) & =f\left(y+t Y_{i}\right) \\
\left(\rho\left(e^{t Z}\right) f\right)(y) & =\chi(t) f(y) .
\end{aligned}
$$

This representation is irreducible and unitary with respect to the usual inner product of $\mathcal{H}_{\rho}$.

Notation. Henceforth, whenever a locally compact group $N$ is isomorphic to a Heisenberg group, we denote the subset of $\hat{N}$ consisting of the family of the infinite-dimenional representations constructed above by $\hat{N}_{\text {。 }}$.

2.3. The Weil representation. Let $S p\left(W_{n}\right)$ be the symplectic group associated to $W_{n}$. When $\mathbb{F}=\mathbb{C}$, take $M p\left(W_{n}\right)=S p\left(W_{n}\right)$, and otherwise let $M p\left(W_{n}\right)$ be the (metaplectic) double covering of $S p\left(W_{n}\right)$ (see We2]). $M p\left(W_{n}\right)$ acts through $S p\left(W_{n}\right)$ on $H_{n} \approx \exp \left(\mathfrak{h}_{n}\right)$ (or equivalently on $\mathfrak{h}_{n}$ ) as follows. For any $w \oplus z \in W_{n} \oplus \mathfrak{z}$,

$$
g: w \oplus z \rightarrow g \cdot w \oplus z .
$$

One can consider the semidirect product $M p\left(W_{n}\right) \ltimes H_{n}$. We have (see We2]):

Proposition 2.3.1. Let $\chi$ be any nontrivial additive character of $\mathbb{F}$. Then the irreducible unitary representation $\rho$ of $H_{n}$ with central character $\chi$ can be extended to a unitary representation (still denoted by $\rho$ ) of $M p\left(W_{n}\right) \ltimes H_{n}$; i.e. for any $g \in M p\left(W_{n}\right)$ and any $h \in H_{n}$ :

$$
\rho(g) \rho(h) \rho\left(g^{-1}\right)=\rho\left(g h g^{-1}\right)
$$

\section{Construction of H-tower groups}

3.1. The Heisenberg parabolic. We use the notation of section 1.2 , Let $\tilde{\beta}$ be the highest root in $\Delta$ with respect to the positive system chosen in section 1.2 and let $H_{\tilde{\beta}}$ be the coroot for $\tilde{\beta}$. Let $(\cdot, \cdot)$ denote the $\mathbf{G}$-invariant symmetric bilinear form introduced in that section; i.e. $(\tilde{\beta}, \tilde{\beta})=2$. One obtains a grading

$$
\mathfrak{g}=\bigoplus_{j \in \mathbb{Z}} \mathfrak{g}_{j}
$$

where $\mathfrak{g}_{j}$ is the $j$-eigenspace of $\operatorname{ad}\left(H_{\tilde{\beta}}\right)$; i.e.

$$
\mathfrak{g}_{j}=\left\{X \in \mathfrak{g}:\left[H_{\tilde{\beta}}, X\right]=j X\right\} .
$$

Note that in fact $\mathfrak{g}_{k}=\{0\}$ for $|k|>2$. The Jacobi identity implies $\left[\mathfrak{g}_{i}, \mathfrak{g}_{j}\right] \subseteq \mathfrak{g}_{i+j}$. Note that $\mathfrak{g}_{1}=\mathfrak{g}_{-1}=\{0\}$ if and only if $\mathfrak{g}=\mathfrak{s l}_{2}$.

In the next proposition and at some points of this paper, we will consider $\mathfrak{g}$ as above such that:

$\diamond \quad \mathfrak{g} \neq \mathfrak{s l}_{2}$ and $\tilde{\beta}$ is defined over $\mathbb{F}$. 
Condition (3.1) is not a crucial assumption. In fact the author believes that it can be removed. See the discussion in the remark after Proposition 3.1.1.

Proposition 3.1.1. Let $\mathfrak{g}$ satisfy condition (3.1). Then

1. $\mathfrak{g}_{0} \oplus \mathfrak{g}_{1} \oplus \mathfrak{g}_{2}$ is a parabolic subalgebra, $\mathfrak{g}_{1} \oplus \mathfrak{g}_{2}$ is its nilradical, and

$$
\left[\left[\mathfrak{g}_{0}, \mathfrak{g}_{0}\right], \mathfrak{g}_{2}\right]=\{0\} .
$$

The nilradical is a Heisenberg Lie algebra with center $\mathfrak{g}_{2}$ and symplectic space $\mathfrak{g}_{1}$. One can describe the symplectic form $<\cdot, \cdot>_{1}$ on $\mathfrak{g}_{1}$ using the Lie bracket as follows: fix $X_{2} \in \mathfrak{g}_{2}-\{0\}$ and let $<\cdot, \cdot>_{1}$ be such that

$$
\text { for every } X, Y \in \mathfrak{g}_{1}, \quad[X, Y]=<X, Y>_{1} \cdot X_{2} .
$$

Then $\langle\cdot, \cdot\rangle_{1}$ will be nondegenerate, and the (adjoint) representation of $\left[\mathfrak{g}_{0}, \mathfrak{g}_{0}\right]$ on $\mathfrak{g}_{1}$ will be a symplectic representation with respect to $\left.<\cdot, \cdot\right\rangle_{1}$.

2. When $\mathfrak{g}$ is not of type $\mathbf{A}_{l}(l>1)$, the parabolic of part 1 is characterized by the subset $\Delta_{B}-\{\beta\}$ of $\Delta_{B}$ for the unique simple root $\beta \in \Delta_{B}$ which satisfies $(\beta, \tilde{\beta}) \neq 0$. For type $\mathbf{A}_{l}(l>1)$, there are two such simple roots $\beta^{\prime}, \beta^{\prime \prime}$, and the parabolic corresponds to $\Delta_{B}-\left\{\beta^{\prime}, \beta^{\prime \prime}\right\}$.

3. When $\mathfrak{g}$ is not of type $\mathbf{A}_{l}(l>1), \beta$ corresponds to a simple restricted root (still denoted by $\beta)$. For $\mathfrak{g}$ of type $\mathbf{A}_{l}(l>2)$ and $\mathfrak{g}_{\mathbb{F}}$ non-split, the pair $\left\{\beta^{\prime}, \beta^{\prime \prime}\right\}$ corresponds to a simple restricted root $\beta$ in the restricted root system.

Let the sets $S \subset \Delta_{B}$ and $T \subset \Sigma_{B}$ be defined as follows. Set $S=\{\beta\}$ when $\mathfrak{g}$ is not of type $\mathbf{A}_{l}(l>1)$ and $S=\left\{\beta^{\prime}, \beta^{\prime \prime}\right\}$ otherwise. Also, set $T=\left\{\beta^{\prime}, \beta^{\prime \prime}\right\}$ when $\mathfrak{g}_{\mathbb{F}}$ is split and $\mathfrak{g}$ is of type $\mathbf{A}_{l}(l>1)$, and $T=\{\beta\}$ otherwise.

4. The parabolic $\mathfrak{p}_{\Delta_{B}-S}$ is defined over $\mathbb{F}$, and $\left(\mathfrak{p}_{\Delta_{B}-S}\right)_{\mathbb{F}}=\mathfrak{p}_{\Sigma_{B}-T}$. Its nilradical is a Heisenberg algebra. $\mathfrak{p}_{\Delta_{B}-S}$ is a maximal parabolic when $\mathfrak{g}$ is not of type $\mathbf{A}_{l}$, $(l>1)$.

Proof. See [GW, §2] and [Tor, §10].

Remark. The assumption that $\mathfrak{g}$ satisfies condition (3.1) holds for all but a very small class of Lie algebras $\mathfrak{g}$. Fox example, when $\mathbb{F}=\mathbb{R}$, the cases where $\tilde{\beta}$ is not defined over $\mathbb{R}$ are $\mathfrak{f}_{4(-20)}, \mathfrak{e}_{6(-26)}, \mathfrak{s p}(p, q)$, and $\mathfrak{s l}(n, \mathbb{H})$. The notion of rank in this paper is based on the existence of a unipotent subgroup of $G$ which is expressible as a tower of extensions by Heisenberg groups (see Definition [3.2.5), and therefore it is not applicable to the groups mentioned above. However, in all of these groups there does exist a similar structure which is called an OKP subgroup in [HRW]. Therefore in principle the main results of this paper (especially Theorem 5.3.2) should generalize to groups associated with these real forms. However, addressing the technical problems which arise with including those cases in this paper makes it much more technical. To keep our presentation as simple and uniform as possible, we do not include those special cases in this paper. The author intends to deal with those cases elsewhere. 
Definition 3.1.2. The parabolic subalgebras $\mathfrak{p}_{\Delta_{B}-S}$ of $\mathfrak{g}$ and $\mathfrak{p}_{\Sigma_{B}-T}$ of $\mathfrak{g}_{\mathbb{F}}$ or their corresponding parabolic subgroups $\mathbf{P}_{\Delta_{B}-S}$ of $\mathbf{G}$ and $P_{\Sigma_{B}-T}=\left(\mathbf{P}_{\Delta_{B}-S}\right)_{\mathbb{F}}$ of $\mathbf{G}_{\mathbb{F}}$ (or the parabolic of $G$ which corresponds to $P_{\Sigma_{B}-T}$ ) are called the Heisenberg parabolics.

We tend to drop their identifying subscripts for simplicity when there is no risk of confusion.

Table I below demonstrates the structure of $\mathfrak{g}_{1}$ as a representation of $\left[\mathfrak{g}_{0}, \mathfrak{g}_{0}\right]$. Here $V_{\varpi}$ denotes the representation with highest weight $\varpi$ and $\varpi_{i}$ denotes the $i$-th fundamental weight of the corresponding Lie algebra. We use the notation of [Bou, Planche I] for numbering fundamental weights. See also [Ti] for more explicit information.

\begin{tabular}{|l|c|c|}
\hline $\mathfrak{g}$ & {$\left[\mathfrak{g}_{0}, \mathfrak{g}_{0}\right]$} & $\mathfrak{g}_{1}$ \\
\hline $\mathbf{A}_{\mathbf{l}}(\mathbf{l} \geq 3)$ & $\mathbf{A}_{\mathbf{l}-2}$ & $V_{\varpi_{1}} \oplus V_{\varpi_{1}}^{*}$ \\
$\mathbf{B}_{\mathbf{2}}$ & $\mathbf{A}_{\mathbf{1}}$ & $V_{\varpi_{1}}$ \\
$\mathbf{B}_{\mathbf{3}}$ & $\mathbf{A}_{\mathbf{1}} \times \mathbf{A}_{\mathbf{1}}$ & $V_{\varpi_{1}} \hat{\otimes} V_{\varpi_{1}}$ \\
$\mathbf{B}_{\mathbf{1}}(\mathbf{l} \geq 4)$ & $\mathbf{A}_{\mathbf{1}} \times \mathbf{B}_{\mathbf{l}-2}$ & $V_{\varpi_{1}} \hat{\otimes} V_{\varpi_{1}}$ \\
$\mathbf{C}_{\mathbf{2}}$ & $\mathbf{A}_{\mathbf{1}}$ & $V_{\varpi_{1}}$ \\
$\mathbf{C}_{\mathbf{l}}(\mathbf{l} \geq 3)$ & $\mathbf{C}_{\mathbf{l}-\mathbf{1}}$ & $V_{\varpi_{1}}$ \\
$\mathbf{D}_{\mathbf{4}}$ & $\mathbf{A}_{\mathbf{1}} \times \mathbf{A}_{\mathbf{1}} \times \mathbf{A}_{\mathbf{1}}$ & $V_{\varpi_{1}} \hat{\otimes} V_{\varpi_{1}} \hat{\otimes} V_{\varpi_{1}}$ \\
$\mathbf{D}_{\mathbf{5}}$ & $\mathbf{A}_{\mathbf{1}} \times \mathbf{A}_{\mathbf{3}}$ & $V_{\varpi_{1}} \hat{\otimes} V_{\varpi_{2}}$ \\
$\mathbf{D}_{\mathbf{1}}(\mathbf{l} \geq 6)$ & $\mathbf{A}_{\mathbf{1}} \times \mathbf{D}_{\mathbf{l}-\mathbf{2}}$ & $V_{\varpi_{1}} \hat{\otimes} V_{\varpi_{1}}$ \\
$\mathbf{E}_{\mathbf{6}}$ & $\mathbf{A}_{\mathbf{5}}$ & $V_{\varpi_{3}}$ \\
$\mathbf{E}_{\mathbf{7}}$ & $\mathbf{D}_{\mathbf{6}}$ & $V_{\varpi_{6}}$ \\
$\mathbf{E}_{\mathbf{8}}$ & $\mathbf{E}_{\mathbf{7}}$ & $V_{\varpi_{7}}$ \\
$\mathbf{F}_{\mathbf{4}}$ & $\mathbf{C}_{\mathbf{3}}$ & $V_{\varpi_{3}}$ \\
$\mathbf{G}_{\mathbf{2}}$ & $\mathbf{A}_{\mathbf{1}}$ & $V_{3 \varpi_{1}}$ \\
\hline
\end{tabular}

Table I

3.2. The H-tower subgroup $N_{\Gamma}$ of $G$. Let $\mathfrak{g}$ be as in condition (3.1). In this section we describe a nilpotent subalgebra of $\mathfrak{g}$ (and another one of $\mathfrak{g}_{\mathbb{F}}$ ) which is fundamental to our definition of rank. The construction of these nilpotent subalgebras is based on what is usually referred to as Kostant's Cascade. We show that this nilpotent Lie subalgebra is actually the nilradical of a parabolic subalgebra (see Propositions 3.2.3 and 3.2.4). The unipotent subgroup of $G$ which corresponds to this nilpotent subalgebra will play an important role in the rest of the paper.

First assume that $\mathfrak{g}$ splits over $\mathbb{F}$; i.e. all roots of $\mathfrak{g}$ are defined over $\mathbb{F}$. Let $\mathfrak{p}=\mathfrak{l} \oplus \mathfrak{n}$ be the Heisenberg parabolic of $\mathfrak{g}$, obtained by Proposition 3.1.1 with the usual Levi decomposition; i.e. $\mathfrak{l}$ is the Levi factor and $\mathfrak{n}$ is the nilradical of $\mathfrak{p}$. In the case of orthogonal algebras the commutator $[\mathfrak{l}, \mathfrak{l}]$ is not a simple Lie algebra. In fact when $\mathfrak{g}$ is of types $\mathbf{B}_{l}(l \geq 3)$ or $\mathbf{D}_{l}(l>4)$, the commutator $[\mathfrak{l}, \mathfrak{l}]$ is a direct sum of the form

$$
[\mathfrak{l}, \mathfrak{l}]=\mathfrak{s l}_{2} \oplus \mathfrak{s}
$$

where $\mathfrak{s}$ is simple. When $\mathfrak{g}$ is of type $\mathbf{D}_{4}$, we have

$$
[\mathfrak{l}, \mathfrak{l}]=\mathfrak{s l}_{2} \oplus \mathfrak{s l}_{2} \oplus \mathfrak{s l}_{2} .
$$


Definition 3.2.1. Let $\mathfrak{m}$ be defined as follows:

I. If $\mathfrak{g}$ is of types $\mathbf{D}_{l}(l>4)$ or $\mathbf{B}_{l}(l>3)$ then $\mathfrak{m}$ is equal to the summand $\mathfrak{s}$ given in (3.2).

II. If $\mathfrak{g}$ is of types $\mathbf{A}_{2}, \mathbf{A}_{3}, \mathbf{B}_{2}=\mathbf{C}_{2}, \mathbf{B}_{3}, \mathbf{D}_{4}$ or $\mathbf{G}_{2}$ then $\mathfrak{m}=\{0\}$.

III. Otherwise, $\mathfrak{m}=[\mathfrak{l}, \mathfrak{l}]$.

One can repeatedly apply Proposition 3.1 .1 as follows. First we apply it to $\mathfrak{m}$. If $\mathfrak{m}$ is nonzero, then $\mathfrak{m} \neq \mathfrak{s l}_{2}$ and Proposition 3.1.1 guarantees the existence of a Heisenberg parabolic in $\mathfrak{m}$. Let $\mathfrak{p}^{\prime}$ be this parabolic of $\mathfrak{m}$, and let $\mathfrak{l}^{\prime}$ be the Levi factor of $\mathfrak{p}^{\prime}$. Let $\mathfrak{m}^{\prime}$ be the subalgebra of $\left[\mathfrak{l}^{\prime}, \mathfrak{l}^{\prime}\right]$ which is defined in the same way that $\mathfrak{m}$ was defined as a subalgebra of $[\mathfrak{l}, \mathfrak{l}]$ in Definition [3.2.1. Then we apply Proposition 3.1.1] to $\mathrm{m}^{\prime}$, and so on. This process can be repeated as long as Proposition 3.1.1 can be applied. As a result, we obtain a sequence $S_{1}, \ldots, S_{r}$ of subsets of $\Delta_{B}$, where each $S_{i}$, defined as in part 2 of Proposition 3.1.1 contains either a simple root or a pair of simple roots. Each $S_{i}$ corresponds to the "highest root" $\tilde{\beta}_{i}$ obtained in the $i$-th step. We also denote the sequence of Heisenberg parabolics by $\mathfrak{p}^{1}, \ldots, \mathfrak{p}^{r}$, with the Levi decomposition

$$
\mathfrak{p}^{j}=\mathfrak{l}^{j} \oplus \mathfrak{n}^{j} .
$$

Therefore, $\mathfrak{p}^{1}$ is the Heisenberg parabolic of $\mathfrak{g}, \mathfrak{p}^{2}$ is the Heisenberg parabolic of $\mathfrak{m}$, and so on. Each $\mathfrak{n}^{j}$ is normalized by any $\mathfrak{l}^{j^{\prime}}$ for $j^{\prime} \geq j$ and hence by $\mathfrak{n}^{j^{\prime}}$. For a similar reason, $\mathfrak{n}^{j^{\prime}}$ acts trivially on the center of $\mathfrak{n}^{j}$. Each $\mathfrak{n}^{j}$ is isomorphic to a Heisenberg algebra $\mathfrak{h}_{d_{i}}$ for some $d_{i}$. Therefore the Lie algebra $\mathfrak{n}^{1} \oplus \cdots \oplus \mathfrak{n}^{r}$ is a tower of successive extensions by Heisenberg $\overline{\mathbb{F}}$-algebras. The following proposition is obvious.

Proposition 3.2.2. $\mathfrak{n}^{1} \oplus \cdots \oplus \mathfrak{n}^{r}$ is equal to the nilradical of the parabolic subalgebra $\mathfrak{p}_{\Gamma}$ of $\mathfrak{g}$ where

$$
\Gamma=\Delta_{B}-\left(S_{1} \cup \ldots \cup S_{r}\right) .
$$

Now assume $\mathfrak{g}$ satisfies condition (3.1) but it is not necessarily $\mathbb{F}$-split. Recall that the positive system for $\Sigma$ is compatible with the one for $\Delta$. We can apply Proposition 3.1.1 repeatedly again. Note that the number of possible iterations for a non-split $\mathbb{F}$-form of $\mathfrak{g}$ is often smaller than the number of possible iterations in the split case. (In certain groups with small rank, they may be equal.) This is because the successive Levi factors may fail to satisfy condition (3.1).

Part 3 of Proposition 3.1 .1 yields a sequence $T_{1}, \ldots, T_{s}$ of subsets of $\Sigma_{B}$ (for some $s \leq r$ ) where each $T_{j}$ contains a simple restricted root or a pair of them. Again we obtain a nested sequence

$$
\mathfrak{p}^{1} \supset \cdots \supset \mathfrak{p}^{s}
$$

of Heisenberg parabolics (this time inside $\mathfrak{g}_{\mathbb{F}}$ ) and the nilradical of any $\mathfrak{p}^{i}$ is normalized by the nilradical of any $\mathfrak{p}^{i^{\prime}}$ when $i<i^{\prime}$. Therefore $\mathfrak{p}^{1}$ is the Heisenberg parabolic subalgebra of $\mathfrak{g}_{\mathbb{F}}$. We warn the reader that the new $\mathfrak{p}^{i}$ 's are different from those which appear immediately before Proposition [3.2.2, in fact the older $\mathfrak{p}^{i}$ s are obtained from the newer $\mathfrak{p}^{i}$ s by an extension of scalars. 


\begin{tabular}{|c|c|c|c|c|c|}
\hline $\mathfrak{g}_{\mathbb{F}}$ & $\mathfrak{m}_{\mathbb{F}}$ & $s$ & $\mathfrak{g}_{\mathbb{F}}$ & $\mathfrak{m}_{\mathbb{F}}$ & $s$ \\
\hline$A_{2,2}$ & - & 1 & $A_{3,3}$ & - & 1 \\
\hline$A_{r, r} \quad(r \geq 4)$ & $A_{r-2, r-2}$ & $\left|\frac{r}{2}\right|$ & ${ }^{2} A_{32}^{(1)}$ & - & 1 \\
\hline${ }^{2} A_{2 r-1, r}^{(1)} \quad(r \geq 3)$ & ${ }^{2} A_{2 r-3 r-1}^{(1)}$ & $r-1$ & ${ }^{2} A_{2,1}^{(1)}$ & - & 1 \\
\hline${ }^{2} A_{2 r, r}^{(1)} \quad(r \geq 2)$ & ${ }^{2} A_{2 r-2, r-1}^{(1)}$ & 8 & ${ }^{2} A_{3,1}^{(1)}$ & - & 1 \\
\hline${ }^{2} A_{2 r+1, r}^{(1)} \quad(r \geq 2)$ & ${ }^{2} A_{2 r-1, r-1}^{(1)}$ & $r$ & $B_{3,3}$ & - & 1 \\
\hline$B_{4,4}$ & $C_{2,2}$ & 2 & $(r \geq 4)$ & $B_{r-2, r-2}$ & $\left\lfloor\frac{r}{2}\right\rfloor$ \\
\hline$B_{3,2}$ & - & 1 & $B_{4,3}$ & - & 1 \\
\hline$(r \geq 5)$ & $B_{r-2, r-3}$ & $\left\lfloor\frac{r-1}{2}\right\rfloor$ & $C_{2,2}$ & - & 1 \\
\hline$C_{r, r} \quad(r \geq 3)$ & $C_{r-1, r-1}$ & $r-1$ & ${ }^{1} D_{44}^{(1)}$ & - & 1 \\
\hline${ }^{1} D_{5,5}^{(1)}$ & $A_{3,3}$ & 2 & ${ }^{1} D_{r, r}^{(1)}$ & ${ }^{1} D_{r-2, r-2}^{(1)}$ & $\left\lfloor\frac{r-1}{2}\right\rfloor$ \\
\hline${ }^{1} D_{4,2}^{(1)}$ & - & 1 & ${ }^{1} D_{5,3}^{(1)}$ & $-r-2, r-2$ & $\begin{array}{ll}2 \\
1\end{array}$ \\
\hline${ }^{1} D_{r+2, r}^{(1)} \quad(r \geq 4)$ & ${ }^{1} D_{r, r-2}^{(1)}$ & $\left\lfloor\frac{r}{2}\right\rfloor$ & ${ }^{2} D_{4,3}^{(1)}$ & - & 1 \\
\hline${ }^{2} D_{5,4}^{(1)}$ & ${ }^{2} A_{3,2}^{(1)}$ & 2 & ${ }^{2} D_{r+1, r}^{(1)} \quad(r \geq 5)$ & ${ }^{2} D_{r-1, r-2}^{(1)}$ & $\left\lfloor\frac{r-1}{2}\right\rfloor$ \\
\hline${ }^{1} D_{4,2}^{(2)}$ & - & 1 & ${ }^{1} D_{2 r, r}^{(2)} \quad(r \geq 3)$ & ${ }^{1} D_{2 r-2, r-1}^{(2)}$ & $r-1$ \\
\hline${ }^{1} D_{5,1}^{(2)}$ & - & 1 & ${ }^{1} D_{2 r+3, r}^{(2)} \quad(r \geq 2)$ & ${ }^{1} D_{2 r+1, r-1}^{(2)}$ & $r$ \\
\hline${ }^{2} D_{5,2}^{(2)}$ & ${ }^{2} A_{3,1}^{(1)}$ & 2 & ${ }^{2} D_{2 r+1, r}^{(2)} \quad(r \geq 3)$ & ${ }^{2} D_{2 r-1, r-1}^{(2)}$ & $r$ \\
\hline${ }^{2} D_{4.1}^{(2)}$ & $-{ }^{0,1}$ & 1 & ${ }^{2} D_{2 r+2, r}^{(2)} \quad(r \geq 3)$ & ${ }^{2} D_{2 r r-1}^{(2)}$ & $r$ \\
\hline${ }^{3} D_{4}^{4,1}$ & - & 1 & ${ }^{6} D_{4}^{2 r+2, r}$ & $-\quad 2 r, r-1$ & 1 \\
\hline${ }^{1} E_{6,2}^{16}$ & - & 1 & ${ }^{1} E_{6,6}^{0}$ & $A_{5,5}$ & 3 \\
\hline${ }^{2} E_{6,4}^{2}$ & ${ }^{2} A_{5,3}^{(1)}$ & 3 & $E_{7,4}^{9}$ & ${ }^{1} D_{6.3}^{(2)}$ & 3 \\
\hline$E_{7}^{0}{ }^{0,4}$ & ${ }^{1} D_{66}^{(1)}$ & 3 & $E_{8,8}^{0}$ & $E_{7}^{0}$ & 4 \\
\hline$F_{44}^{0}$ & $C_{3,3}$ & 3 & $G_{22}^{0,8}$ & - & 1 \\
\hline
\end{tabular}

Table II

\begin{tabular}{|c|c|c|c|c|c|c|c|}
\hline $\mathfrak{g}_{\mathbb{F}}$ & & $\mathfrak{m}_{\mathbb{F}}$ & $s$ & $\mathfrak{g}_{\mathbb{F}}$ & & $\mathfrak{m}_{\mathbb{F}}$ & $s$ \\
\hline $\mathfrak{s l}_{3}(\mathbb{R})$ & & - & 1 & $\mathfrak{s l}_{4}(\mathbb{R})$ & & - & 1 \\
\hline $\mathfrak{s l}_{n}(\mathbb{R})$ & $(n \geq 5)$ & $\mathfrak{s l}_{n-2}(\mathbb{R})$ & $\left\lfloor\frac{n-1}{2}\right\rfloor$ & $\mathfrak{s u}(1, q)$ & $(q>1)$ & - & 1 \\
\hline $\mathfrak{s u}(2,2)$ & & - & 1 & $\mathfrak{s u}(r, q)$ & $(2 \leq r<q)$ & $\mathfrak{s u}(r-1, q-1)$ & $r$ \\
\hline $\mathfrak{s u}(q, q)$ & $(3 \leq q)$ & $\mathfrak{s u}(q-1, q-1)$ & $q-1$ & $\mathfrak{s o}(1, q)$ & $(q \geq 4)$ & - & 1 \\
\hline $\mathfrak{s o}(2, q)$ & $(q \geq 3)$ & - & 1 & $\mathfrak{s o}(3, q)$ & $(q \geq 3)$ & - & 1 \\
\hline $\mathfrak{s o}(4,4)$ & & - & 1 & $\mathfrak{s o}(4, q)$ & $(q \geq 5)$ & $\mathfrak{s o}(2, q-2)$ & 2 \\
\hline $\mathfrak{s o}(r, q)$ & $(5 \leq r<q)$ & $\mathfrak{s o}(r-2, q-2)$ & $\left\lfloor\frac{r}{2}\right\rfloor$ & $\mathfrak{s o}(q, q)$ & $(5 \leq q)$ & $\mathfrak{s o}(q-2, q-2)$ & $\left\lfloor\frac{q-1}{2}\right\rfloor$ \\
\hline $\mathfrak{s p}_{4}(\mathbb{R})$ & & - & 1 & $\mathfrak{s p}_{2 n}(\mathbb{R})$ & $(n \geq 3)$ & $\mathfrak{s p}_{2 n-2}(\mathbb{R})$ & $n-1$ \\
\hline $\mathfrak{s o}^{*}(6)$ & & - & 1 & $\mathfrak{s o}^{*}(8)$ & & & 1 \\
\hline $\mathfrak{s o}^{*}(2 r)$ & $(r \geq 5)$ & $\mathfrak{s o}^{*}(2 r-4)$ & $\left\lfloor\frac{r-1}{2}\right\rfloor$ & $\left(\mathfrak{e}_{6}, \mathfrak{s p}_{4}\right)$ & & $\mathfrak{s l}_{6}(\mathbb{R})$ & 3 \\
\hline$\left(\mathfrak{e}_{6}, \mathfrak{s u}_{6} \times \mathfrak{s u}_{2}\right)$ & & $\mathfrak{s u}(3,3)$ & 3 & $\left(\mathfrak{e}_{6}, \mathfrak{s o}(10) \times \mathfrak{u}(1)\right)$ & & $\mathfrak{s u}(1,5)$ & 2 \\
\hline$\left(\mathfrak{e}_{7}, \mathfrak{s u}_{8}\right)$ & & $\mathfrak{s o}(6,6)$ & 3 & $\left(\mathfrak{e}_{7}, \mathfrak{s o}(12) \times \mathfrak{s u}_{2}\right)$ & & $\mathfrak{s o}^{*}(12)$ & 3 \\
\hline$\left(\mathfrak{e}_{7}, \mathfrak{e}_{6} \times \mathfrak{u}(1)\right)$ & & $\mathfrak{s o}(2,10)$ & 2 & $\left(\mathfrak{e}_{8}, \mathfrak{s o}(12)\right)$ & & $\left(\mathfrak{e}_{7}, \mathfrak{s u _ { 8 } )}\right.$ & 4 \\
\hline$\left(\mathfrak{e}_{8}, \mathfrak{e}_{7} \times \mathfrak{s u}_{2}\right)$ & & $\left(\mathfrak{e}_{7}, \mathfrak{e}_{6} \times \mathfrak{u}(1)\right)$ & 3 & $\left(\mathfrak{f}_{4}, \mathfrak{s p}_{3} \times \mathfrak{s u}_{2}\right)$ & & $\mathfrak{s p}_{6}(\mathbb{R})$ & 3 \\
\hline$\left(\mathfrak{g}_{2}, \mathfrak{s u}_{2} \times \mathfrak{s u}_{2}\right)$ & & - & 1 & & & & \\
\hline
\end{tabular}


Tables II and III explain how Proposition 3.1.1 is applied iteratively to $\mathfrak{g}_{\mathbb{F}}$. For simplicity, the real and the $p$-adic cases are separated: Table II is for the $p$-adic case and Table III is for the real case.

The column $\mathfrak{g}_{\mathbb{F}}$ in Table II shows the Tits index of $\mathfrak{g}_{\mathbb{F}}$ and the column $\mathfrak{m}_{\mathbb{F}}$ shows the Tits index of $\mathfrak{m}_{\mathbb{F}}$. For any $\mathfrak{g}_{\mathbb{F}}$ the number $s$ is given too. Real exceptional Lie algebras in Table III are identified by the symmetric pairs $\left(\mathfrak{g}_{\mathbb{R}}, \mathfrak{k}_{\mathbb{R}}\right)$. The only $\mathbb{F}$-forms of $\mathfrak{g}$ which appear in the columns $\mathfrak{g}_{\mathbb{F}}$ of both of the tables are those for which $\mathfrak{g}$ satisfies condition (3.1). If an entry in the column corresponding to $\mathfrak{m}_{\mathbb{F}}$ is equal to "-", this means either that the Lie algebra $\mathfrak{m}$ is equal to $\{0\}$ or that the Lie algebra $\mathfrak{m}$ is semisimple but it does not satisfy condition (3.1), i.e. the highest root of $\mathfrak{m}$ is not defined over $\mathbb{F}$. Therefore one can use Tables II and III to see how many times Proposition 3.1.1 can be applied to a particular $\mathbb{F}$-form of $\mathfrak{g}$.

We denote the nilradical of the parabolic $\mathfrak{p}^{j}$ of $\mathfrak{g}_{\mathbb{F}}$ by $\mathfrak{h}^{j}$. It is an $\mathbb{F}$-Heisenberg algebra.

Proposition 3.2.3. The nilpotent Lie subalgebra $\mathfrak{h}^{1} \oplus \cdots \oplus \mathfrak{h}^{s}$ of $\mathfrak{g}_{\mathbb{F}}$ is equal to the nilradical of the parabolic subalgebra $\mathfrak{p}_{\Gamma}$ of $\mathfrak{g}_{\mathbb{F}}$ where

$$
\Gamma=\Sigma_{B}-\left(T_{1} \cup \ldots \cup T_{s}\right) .
$$

Moreover, we have the following proposition, which describes the relationship between $\mathfrak{n}^{i}$ s and $\mathfrak{h}^{i}$ 's.

Proposition 3.2.4. Let $\mathfrak{p}_{\Gamma}$ be the parabolic subalgebra of $\mathfrak{g}_{\mathbb{F}}$ defined in Proposition 3.2 .3 with Levi decomposition $\mathfrak{p}_{\Gamma}=\mathfrak{l}_{\Gamma} \oplus \mathfrak{n}_{\Gamma}$. Then

$$
\mathfrak{n}_{\Gamma} \otimes \overline{\mathbb{F}}=\mathfrak{n}^{1} \oplus \cdots \oplus \mathfrak{n}^{s}
$$

(where the $\mathfrak{n}^{j}$ 's are the same as those which appear in Proposition 3.2 .2$)$ and thus $\mathfrak{n}_{\Gamma} \otimes \overline{\mathbb{F}}$ is equal to the nilradical of the parabolic $\mathfrak{p}_{\Gamma^{\prime}}$ of $\mathfrak{g}$ where

$$
\Gamma^{\prime}=\Delta_{B}-\left(S_{1} \cup \ldots \cup S_{s}\right) .
$$

Therefore $\mathbf{P}_{\Gamma^{\prime}}$ is an $\mathbb{F}$-parabolic of $\mathbf{G}$ and $\left(\mathbf{P}_{\Gamma^{\prime}}\right)_{\mathbb{F}}=P_{\Gamma}$, where $P_{\Gamma}$ is the parabolic of $G$ which is associated to the set $\Gamma \subseteq \Sigma_{B}$ defined in Proposition 3.2.3. $P_{\Gamma}$ will play a significant role in the rest of the paper. Its Levi decomposition can be written as

$$
P_{\Gamma}=L_{\Gamma} N_{\Gamma}
$$

Definition 3.2.5. Let $U$ be the group of $\mathbb{F}$-points of a unipotent linear algebraic $\mathbb{F}$-group U. $U$ is said to be an "H-tower" group if and only if it satisfies one of the properties I or II below.

I. $U=\{1\}$; i.e. $U$ is trivial.

II. $U$ is isomorphic to a semidirect product $U^{\prime} \ltimes U^{\prime \prime}$ where

i. $U^{\prime \prime}$ is an $\mathbb{F}$-Heisenberg group and is the group of $\mathbb{F}$-points of an algebraic $\mathbb{F}$-subgroup $\mathbf{U}^{\prime \prime}$ of $\mathbf{U}$.

ii. $U^{\prime}$ is the group of $\mathbb{F}$-rational points of an algebraic subgroup $\mathbf{U}^{\prime}$ of $\mathbf{U}$ and the action of $U^{\prime}$ on $U^{\prime \prime}$ in the semidirect product $U^{\prime} \ltimes U^{\prime \prime}$ comes from an algebraic action (defined over $\mathbb{F}$ ) of $\mathbf{U}^{\prime}$ on $\mathbf{U}^{\prime \prime}$ by group automorphisms of $\mathbf{U}^{\prime \prime}$ (which, 
a fortiori, leave elements of $\mathcal{Z}\left(\mathbf{U}^{\prime \prime}\right)$ invariant and act on the symplectic space $\mathbf{U}^{\prime \prime} / \mathcal{Z}\left(\mathbf{U}^{\prime \prime}\right)$ via symplectic operators $)^{2}$.

iii. $U^{\prime}$ is an H-tower group.

Therefore an H-tower group $U$ can be expressed as a tower of successive extensions:

$$
U=H^{1} \cdot H^{2} \cdots H^{t}=H^{1} \rtimes\left(\cdots \rtimes\left(H^{t-1} \rtimes H^{t}\right) \cdots\right)
$$

where each $H^{j}$ is a Heisenberg group; i.e. $H^{j}=H_{d_{j}}$ for some $d_{j}$ (see section 2.1).

\section{Remarks.}

1. The name "H-tower" is chosen so that it reminds the reader that the group is a tower of extensions by Heisenberg groups.

2. Real H-tower groups form a subclass of OKP groups defined in [HRW].

3. The number $t$ in (3.5) is referred to as the height of the tower of extensions. It will be denoted by $h t(U)$.

4. Consider an H-tower group $U$ which is expressed as in (3.5). for any $j \in\{1, \ldots, h t(U)\}$ we denote the quotient group $H^{j} \cdots H^{\mathrm{ht}(U)} \approx U / H^{1} \cdots H^{j-1}$ by $U_{j} . U_{j}$ is also an H-tower group.

If in (3.5) we take $U=N_{\Gamma}$ and $H^{j}=\exp \mathfrak{h}^{j}$, then by Proposition 3.2.3 we have

Proposition 3.2.6. $N_{\Gamma}$ is an H-tower group and $\mathrm{ht}\left(N_{\Gamma}\right)=s$, where $s$ is as in Proposition 3.2.3.

\section{RANKABlE REPRESEntations OF H-TOWER GROUPS}

4.1. Oscillator extension and rankable representations. Fix an H-tower group $U$, expressed as a tower of successive extensions as in (3.5). Let $\chi_{1}$ be a nontrivial additive character of $\mathbb{F}$, and consider the unitary representation $\rho_{1}$ of $H^{1}$ with central character $\chi_{1}$ (see section 2.2). When restricted to the inverse image (in the metaplectic group) of the maximal unipotent subgroup of the symplectic group, the Weil representation factors through a representation of the maximal unipotent subgroup of the symplectic group. (In fact the two-fold central extension of the symplectic group splits over the maximal unipotent subgroup and, more importantly, this splitting is unique. This holds because any two splittings would differ by a finite-order character, whereas the maximal unipotent subgroup is a divisible group and therefore it does not have such characters.) Therefore, any representation $\rho$ of any Heisenberg group $H_{n}$ with central character $\chi$ (see section 2.2) is extendable to the unipotent radical of any Borel subgroup of the symplectic group $S p\left(W_{n}\right)$. This implies that the representation $\rho_{1}$ of $H^{1}$ is extendable (in at least one way) to $U$. We still denote this extension by $\rho_{1}$. Note that the extension of $\rho_{1}$ is not necessarily unique, as one can for example twist it by a one dimensional representation of $U$ which is trivial on $H^{1}$. However, since the extension of $\rho$ to the metaplectic group is unique, we can distinguish the extension of $\rho_{1}$ obtained by the restriction of the Weil

\footnotetext{
${ }^{2}$ This means that there is an $\mathbb{F}$-homomorphism of algebraic groups $\Phi: \mathbf{U}^{\prime} \mapsto \mathbf{S p}\left(\mathbf{U}^{\prime \prime} / \mathcal{Z}\left(\mathbf{U}^{\prime \prime}\right)\right)$ and consequently $\Phi\left(U^{\prime}\right) \subseteq S p\left(U^{\prime \prime} / \mathcal{Z}\left(U^{\prime \prime}\right)\right)$.
} 
representation without ambiguity. Let $\rho_{1}$ mean this specific extension.

In a similar fashion, for any $j>1$ one can construct a representation of

$$
U_{j}=U / H^{1} \cdots H^{j-1} \approx H^{j} \cdots H^{\mathrm{ht}(U)}
$$

as follows. We take an arbitrary nontrivial additive character $\chi_{j}$ of $\mathbb{F}$, and the representation $\rho_{j}$ of $H^{j}$ with central character $\chi_{j}$. As before, we use the Weil representation to extend $\rho_{j}$ to $U_{j}$. Since

$$
U_{j} \approx U / H^{1} \cdots H^{j-1}
$$

this representation can be extended to $U$, trivially on $H^{1} \cdots H^{j-1}$. Thus using $\rho_{j}$ we have constructed a representation of $U$. We still keep the notation $\rho_{j}$ for this representation.

Definition 4.1.1. Let $U$ be an H-tower group described as in (3.5). A representation of $U$ is called "rankable" if and only if it is unitarily equivalent to a tensor product of the form

$$
\rho_{1} \otimes \rho_{2} \otimes \cdots \otimes \rho_{k}
$$

for some $k \leq \operatorname{ht}(U)$, where $\rho_{j}$ is the representation of $U$ which is obtained (using the Weil representation) by extending the irreducible representation of $H^{j}$ with central character $\chi_{j}$. The rank of a $k$-fold tensor product (including the case $k=0$, i.e. the trivial representation) is defined to be $k$. If $\sigma$ is a rankable representation of rank $k$, we write $\operatorname{rank}(\sigma)=k$

Remark. It follows from Corollary 4.2.3 that $\operatorname{rank}(\sigma)$ is well-defined; i.e. it is an invariant of the unitary equivalence class of $\sigma$.

4.2. Kirillov theory for rankable representations. We denote the coadjoint orbit attached to an irreducible unitary representation $\sigma$ of a nilpotent group by $\mathcal{O}_{\sigma}^{*}$. (See [Kr1], Kr2, Chapter 3], [G] or [Ki, §2.2] for elaborated treatments of Kirillov's orbital theory.) In his seminal paper [Kr1], Kirillov developed his method of orbits for simply connected nilpotent real Lie groups. However, later in his 1966 ICM lecture he explained that essentially the same theory can be applied to algebraic unipotent groups over $p$-adic fields. See also [Mo1, §4] for more details.

Lemma 4.2.1. Let $N$ be the group of $\mathbb{F}$-rational points of a unipotent linear algebraic $\mathbb{F}$ group $\mathbf{N}$. Let $\sigma$ be an irreducible unitary representation of $N$ and let $\mathcal{O}_{\sigma}^{*}$ be the coadjoint orbit attached to it. Then $\mathcal{O}_{\sigma}^{*}$ is an analytic manifold in the sense of [Se, Chapter III, $\S 2]$.

Proof. The lemma follows immediately from [PR, §3.1, Corollary 2].

Lemma 4.2.1 implies that one can speak of the dimension of a coadjoint orbit. (The transitivity under the group action implies that the dimension is the same around every point.) For any coadjoint orbit $\mathcal{O}^{*}$, let $\operatorname{dim} \mathcal{O}^{*}$ denote its dimension.

Lemma 4.2.2. Let $\mathbf{N}$ be a unipotent algebraic group such that $\mathbf{N}=\mathbf{N}^{\prime} \ltimes \mathbf{N}^{\prime \prime}$ as algebraic $\mathbb{F}$-groups (and the action of $\mathbf{N}^{\prime}$ on $\mathbf{N}^{\prime \prime}$ is defined over $\mathbb{F}$ ) and let $N=N^{\prime} \ltimes N^{\prime \prime}$ be the group of $\mathbb{F}$-rational points of $\mathbf{N}$ (where $N^{\prime}, N^{\prime \prime}$ are the $\mathbb{F}$-points of $\mathbf{N}^{\prime}, \mathbf{N}^{\prime \prime}$ ). Let the Lie algebras of $N^{\prime}, N^{\prime \prime}$ be $\mathfrak{n}^{\prime}, \mathfrak{n}^{\prime \prime}$ respectively. The Lie algebra of the semidirect product $N=N^{\prime} \ltimes N^{\prime \prime}$ is $\mathfrak{n}=\mathfrak{n}^{\prime} \oplus \mathfrak{n}^{\prime \prime}$ as a vector space, and we have a canonical isomorphism of dual spaces 
$\mathfrak{n}^{*}=\mathfrak{n}^{\prime *} \oplus \mathfrak{n}^{\prime \prime *}$. Let $\sigma^{\prime}, \sigma^{\prime \prime}$ be irreducible unitary representations of $N^{\prime}, N^{\prime \prime}$ respectively, such that

1) $\sigma^{\prime}$ is an irreducible unitary representation of $N^{\prime}$, extended trivially on $N^{\prime \prime}$ to a representation $\tilde{\sigma}^{\prime}$ of $N$.

2) $\sigma^{\prime \prime}$ is an irreducible unitary representation of $N^{\prime \prime}$ which extends to a unitary representation $\tilde{\sigma}^{\prime \prime}$ of $N$. (In other words, $\operatorname{Res}_{N^{\prime \prime}}^{N} \tilde{\sigma}^{\prime \prime}=\sigma^{\prime \prime}$.)

Then $\tilde{\sigma}^{\prime} \otimes \tilde{\sigma}^{\prime \prime}$ is an irreducible representation of $N$ and

$$
\operatorname{dim} \mathcal{O}_{\tilde{\sigma}^{\prime} \otimes \tilde{\sigma}^{\prime \prime}}^{*}=\operatorname{dim} \mathcal{O}_{\sigma^{\prime}}^{*}+\operatorname{dim} \mathcal{O}_{\sigma^{\prime \prime}}^{*}
$$

Proof. Consider the map $\mathbf{j}$ defined as the composition

$$
N \mapsto N \times N \mapsto N / N^{\prime \prime} \times N \approx N^{\prime} \times N
$$

where the leftmost map is the diagonal embedding and the middle map (i.e. the map $\left.N \times N \mapsto N / N^{\prime \prime} \times N\right)$ is projection onto the first factor (i.e. it is given by $\left(n_{1}, n_{2}\right) \mapsto$ $\left.\left(n_{1} N^{\prime \prime}, n_{2}\right)\right)$. Observe that

$$
\tilde{\sigma}^{\prime} \otimes \tilde{\sigma}^{\prime \prime}=\operatorname{Res}_{\mathbf{j}(N)}^{N^{\prime} \times N} \sigma^{\prime} \hat{\otimes} \tilde{\sigma}^{\prime \prime}
$$

It is easily seen by Mackey theory Mac1, Theorem 3.12] that $\tilde{\sigma}^{\prime} \otimes \tilde{\sigma}^{\prime \prime}$ is irreducible. Now take a maximal chain of analytic subgroups

$$
N^{\prime} \times N=N^{0} \supset N^{1} \supset N^{2} \supset \cdots \supset \mathbf{j}(N)
$$

such that each $N^{j}$ has codimension 1 in $N^{j-1}$. It follows from (4.1) that if $\sigma^{\prime} \otimes \tilde{\sigma}^{\prime \prime}$ is considered as a representation of $N^{j-1}$, then

$$
\operatorname{Res}_{N^{j}}^{N^{j-1}} \sigma^{\prime} \otimes \tilde{\sigma}^{\prime \prime}
$$

is an irreducible representation of $N^{j}$. Now it follows from the statement of Theorem 2.5.1 of CG that for any such $j$ we are in the situation of part (b) of that theorem. But then Proposition 1.3.4 of [CG] implies that in this case, the dimension of the coadjoint orbit of $\sigma^{\prime} \otimes \tilde{\sigma}^{\prime \prime}$ considered as a representation of $N^{j}$ is the same as the dimension of the coadjoint orbit of $\sigma^{\prime} \otimes \tilde{\sigma}^{\prime \prime}$ considered as a representation of $N^{j-1}$. Consequently,

$$
\operatorname{dim} \mathcal{O}_{\sigma^{\prime} \hat{\otimes} \tilde{\sigma}^{\prime \prime}}^{*}=\operatorname{dim} \mathcal{O}_{\tilde{\sigma}^{\prime} \otimes \tilde{\sigma}^{\prime \prime}}^{*}
$$

Now consider the map q such that

$$
\mathbf{q}: N^{\prime} \times N^{\prime \prime} \mapsto N^{\prime} \times N
$$

which is defined to be the identity map $N^{\prime} \mapsto N^{\prime}$ in the first component and the injection $N^{\prime \prime} \subset N$ in the second component. Then

$$
\operatorname{Res}_{\mathbf{q}\left(N^{\prime} \times N^{\prime \prime}\right)}^{N^{\prime} \times N} \sigma^{\prime} \hat{\otimes} \tilde{\sigma}^{\prime \prime}=\sigma^{\prime} \hat{\otimes} \sigma^{\prime \prime}
$$

which is an irreducible representation. Again a similar argument (taking a maximal chain, etc.) proves that

$$
\operatorname{dim} \mathcal{O}_{\sigma^{\prime} \hat{\otimes} \tilde{\sigma}^{\prime \prime}}^{*}=\operatorname{dim} \mathcal{O}_{\sigma^{\prime} \hat{\otimes} \sigma^{\prime \prime}}^{*}
$$

But the coadjoint orbit $\mathcal{O}_{\sigma^{\prime} \hat{\otimes} \sigma^{\prime \prime}}^{*}$ equals $\mathcal{O}_{\sigma^{\prime}}^{*} \times \mathcal{O}_{\sigma^{\prime \prime}}^{*}$, which completes the proof.

One can apply Lemma 4.2.2 to any H-tower group iteratively and obtain the following result. 
Corollary 4.2.3. Let $U$ be an H-tower group as in (3.5). Let

$$
\sigma=\rho_{1} \otimes \rho_{2} \otimes \cdots \otimes \rho_{k}
$$

be a rankable representation of $U$. (Recall that $\rho_{j}$ is extended from a representation of $H^{j}$ with central character $\chi_{j}$.) Then $\sigma$ is irreducible. Moreover, as an analytic manifold, the coadjoint orbit $\mathcal{O}_{\sigma}^{*}$ has dimension $2\left(n_{1}+\cdots+n_{k}\right)$, where

$$
n_{j}=\frac{\operatorname{dimh}^{j}-1}{2} \text {. }
$$

\section{MAIN THEOREMS}

5.1. Technical issues of central extensions. Let $G, \mathbf{G}, \mathfrak{g}$ be as in section 1.2 such that $\mathfrak{g}$ satisfies condition (3.1). Let $P$ be the Heisenberg parabolic of $G$ (see Definition 3.1.2). Suppose $P=L N$ is the Levi decomposition of $P$. Therefore $N$ is a Heisenberg group. As in section 2.2 let $\rho$ be the irreducible unitary representation of $N$ with an arbitrary nontrivial central character $\chi$. Let $N_{\Gamma}$ be the H-tower subgroup of $G$ constructed in section 3.2 and assume $h t\left(N_{\Gamma}\right)>1$.

This section is mainly devoted to a technical issue which arises with central extensions of $G$. The main result is Proposition 5.1.1. To avoid being distracted from the main point of the paper, the reader may assume Proposition 5.1.1 and go on to section 5.2 .

Proposition 3.1.1 implies that the adjoint action provides a group homomorphism $[L, L] \mapsto S p(N / \mathcal{Z}(N))$. Our next aim is to somehow apply Proposition 2.3.1 and extend $\rho$ to a representation of a larger group which at least contains $N_{\Gamma}$. One natural candidate could be $[P, P]$. However, when $\mathbb{F} \neq \mathbb{C}$, in order to extend the representation $\rho$ of $N$ to $[P, P]$ we need to know that indeed the group homomorphism $[L, L] \mapsto S p(N / \mathcal{Z}(N))$ is a composition of a group homomorphism into the metaplectic group, i.e.

$$
[L, L] \mapsto M p(N / \mathcal{Z}(N))
$$

and the projection map

$$
M p(N / \mathcal{Z}(N)) \mapsto S p(N / \mathcal{Z}(N))
$$

This issue was also dealt with in [Tor] (see [GS, Section 3.3]). We will explain it more clearly below. In fact it can be seen that in some cases, if $\mathbf{P}_{\mathbb{F}}=\mathbf{L}_{\mathbb{F}} \mathbf{N}_{\mathbb{F}}$ is the Heisenberg parabolic of $\mathbf{G}_{\mathbb{F}}$, then $\left[\mathbf{L}_{\mathbb{F}}, \mathbf{L}_{\mathbb{F}}\right]$ does not act as a subgroup of the metaplectic group, and therefore extending $\rho$ to $\left[\mathbf{P}_{\mathbb{F}}, \mathbf{P}_{\mathbb{F}}\right]$ may not be possible. An obvious example is when $\mathbf{G}_{\mathbb{F}}$ is the symplectic group. A less obvious example is the split group of type $\mathbf{F}_{4}$. (Actually, in both cases the extension is possible if we use the metaplectic covers. This may be seen after some simple calculations. For real groups one can use the results of Ad] which tell us when a covering of a real simple Lie group splits over embedded root subgroups $S L_{2}(\mathbb{R})$. However, here we do not rely on such calculations.)

To overcome this difficulty, we will see below that we have to consider an appropriate finite (topological) central extension of $G$ instead of $G$ itself. Note that a representation of $G$ can trivially be considered as a representation of its central extension, and we can always study this extension instead of the original $G$ because the central extension has an H-tower subgroup too, which is identical to $N_{\Gamma}$ and is essentially obtained by 
exponentiating the corresponding nilpotent subalgebra $\mathfrak{n}_{\Gamma}$ of $\mathfrak{g}_{\mathbb{F}}$. This follows from what was explained in section 1.2 as well, that a universal topological central extension of $\mathbf{G}_{\mathbb{F}}$ splits over the maximal unipotent subgroup (see [Du, II 11 Lemme] or [De, Section 1.9]). (In fact the splitting is unique because any two splittings would differ by a finite-order character, whereas any unipotent subgroup is a divisible group and therefore it does not have such characters.) Therefore, as far as it concerns the main results of this work, we can substitute $G$ with such a "good" central extension of $G$, without any loss of generality.

To show the existence of this "good" central extension for an arbitrary $G$, first we show its existence for the group $\mathbf{G}_{\mathbb{F}}$. That is to say, we show that we can find a finite topological central extension $\tilde{\mathbf{G}}_{\mathbb{F}}$ of $\mathbf{G}_{\mathbb{F}}$ in which the representation $\rho$ of $N$ can be extended to a larger subgroup. We warn the reader that we may (and will) think of $N$ as a subgroup of both $\mathbf{G}_{\mathbb{F}}$ and $\tilde{\mathbf{G}}_{\mathbb{F}}$ at the same time, since the latter group has a subgroup identical to the subgroup $N$ of the former one.

Note that once we find $\tilde{\mathbf{G}}_{\mathbb{F}}$, a simple argument implies that the required extension exists for $G$ as well. In fact the existence of a universal topological central extension of $\mathbf{G}_{\mathbb{F}}$ implies that there will be a finite topological central extension which covers both $G$ and $\tilde{\mathbf{G}}_{\mathbb{F}}$. This extension will be the one in which the extension of $\rho$ to a larger subgroup is possible.

Next we show that we can find $\tilde{\mathbf{G}}_{\mathbb{F}}$. Let

$$
1 \longrightarrow F \stackrel{\hat{i}}{\longrightarrow} \tilde{\mathbf{G}}_{\mathbb{F}} \stackrel{\hat{p}}{\longrightarrow} \mathbf{G}_{\mathbb{F}} \longrightarrow 1
$$

be a finite topological central extension of $\mathbf{G}_{\mathbb{F}}$. Let $\mathbf{P}=\mathbf{L N}$ be the Heisenberg parabolic of $\mathbf{G}$ with its usual Levi decomposition. Let $L=\hat{p}^{-1}\left(\mathbf{L}_{\mathbb{F}}\right)$ and let $N$ be the unipotent radical of $P=\hat{p}^{-1}\left(\mathbf{P}_{\mathbb{F}}\right)$. The group $[\mathbf{L}, \mathbf{L}]$, the derived subgroup of $\mathbf{L}$, is a product of isotropic and anisotropic factors (see [Tor, §8.19]). Let $\mathbf{L}^{\text {is }}$ be the isotropic factor of $[\mathbf{L}, \mathbf{L}]$. Let $\mathbf{M}$ be the simply connected subgroup of $[\mathbf{L}, \mathbf{L}]$ whose Lie algebra is equal to $\mathfrak{m}$, where $\mathfrak{m}$ is the Lie algebra introduced in Definition 3.2.1. (The simply connectedness of $\mathbf{M}$ follows from [Tor, $\S 8.19]$.) It follows from $h t\left(N_{\Gamma}\right)>1$ that $\mathbf{M} \subseteq \mathbf{L}^{\text {is }}$. Let $M=\hat{p}^{-1}\left(\mathbf{M}_{\mathbb{F}}\right)$.

Recall that the adjoint action provides a map

$$
M \mapsto S p(N / \mathcal{Z}(N)) .
$$

(See Proposition [3.1.1) Let us call the inverse image of any subgroup $M^{\prime}$ of $M$ inside the metaplectic group $M p(N / \mathcal{Z}(N))$ the metaplectic extension of $M^{\prime}$. It follows from [Tor, $\S 8.25$ and $\S 9.3]$ that if the $\mathbb{F}$-points of the minimal nilpotent $\mathbf{G}$-orbit of $\mathfrak{g}$ contain a $\tilde{\mathbf{G}}_{\mathbb{F}}$-admissible orbit (see [Tor, §3.17] for the definition of an admissible orbit), then there exists a closed subgroup $M^{\prime}$ of $M$, which is a normal subgroup of $L$, such that $\hat{p}\left(M^{\prime}\right) \supseteq \mathbf{M}_{\mathbb{F}}^{+}$(where $\mathbf{M}_{\mathbb{F}}^{+}$is the subgroup of $\mathbf{M}_{\mathbb{F}}$ generated by its $\mathbb{F}$-rational unipotent elements) and the metaplectic extension of $M^{\prime}$ is trivial. (Note that in fact $\hat{p}\left(M^{\prime}\right) \supseteq \mathbf{M}_{\mathbb{F}}$, because by [Tor, §8.19], $\mathbf{M}$ is simply connected and therefore $\mathbf{M}_{\mathbb{F}}^{+}=\mathbf{M}_{\mathbb{F}}$ (see [PR, $\left.\left.\S 7.2\right]\right)$.) This means that one can extend $\rho$ to $M^{\prime} \cdot N$. We keep the notation $\rho$ for such an extension. We will see below that the extension of $\rho$ is actually uniquely determined on a 
slightly smaller group, and therefore using $\rho$ to denote the extension is not ambiguous.

Tables in the end of [Tor] determine which groups $\mathbf{G}_{\mathbb{F}}$ have finite topological central extensions $\tilde{\mathbf{G}}_{\mathbb{F}}$ with admissible orbits. Among all $\mathbb{F}$-forms of groups $\mathbf{G}$ whose Lie algebras $\mathfrak{g}$ satisfy condition (3.1), the only groups which do not have coverings with admissible orbits are groups of Tits index $B_{r, r}$ and $B_{r, r-1}$ in the nonarchimedean case and $S U(p, q)$ and $S O(p, q)$ when $p+q$ is odd in the archimedean case. In these cases, however, the metaplectic extension of $\mathbf{M}_{\mathbb{F}}$ is trivial by [Li2, Lemma 2.2] and [Kaz, Lemma 7]. Therefore if $M^{\prime}=\mathbf{M}_{\mathbb{F}}$ then $\rho$ extends to $M^{\prime}$. This settles the issue of existence of an appropriate covering for $\mathbf{G}_{\mathbb{F}}$.

For a general $G$ we can take $\tilde{G}$ as a finite topological central extension which covers both $G$ and $\tilde{\mathbf{G}}_{\mathbb{F}}$. Let $M_{0}$ be the inverse image of $M^{\prime}$ in $\tilde{G}$. Obviously extending $\rho$ to $M_{0} \cdot N$ is possible. $M_{0}$ has a closed finite-index subgroup $M_{1}$ which is perfect (i.e. $\left.\left[M_{1}, M_{1}\right]=M_{1}\right) . \quad M_{1}$ is found as follows. Define the sequence $M^{(0)}=M_{0}$ and $M^{(i)}=\left[M^{(i-1)}, M^{(i-1)}\right]$. Then each $M^{(i)}$ is a subgroup of $M^{\prime}$ of finite index and the indices are uniformly bounded (in fact one upper bound is the size of the kernel of the map $\left.\tilde{G} \mapsto \mathbf{G}_{\mathbb{F}}\right)$. Therefore there is some $i_{\circ}$ such that $M^{\left(i_{\circ}\right)}=M^{\left(i_{\circ}+1\right)}$; i.e. $M^{(i \circ)}$ is perfect. Let $M_{1}=\overline{M^{\left(i_{\circ}\right)}}$, the closure of $M^{\left(i_{\circ}\right)}$ inside $M^{\prime}$. Then $M_{1}$ is perfect by [Tor, $\$ 8.11$.

The extension of $\rho$ to $M_{1} \cdot N$ is unique. In fact the projective representation of $M_{1}$ is uniquely determined by the relation

$$
\rho(m) \rho(n) \rho\left(m^{-1}\right)=\rho\left(m n m^{-1}\right) \quad \text { for any } \quad n \in N, m \in M_{1}
$$

and since $M_{1}$ is perfect, this projective representation lifts to a linear representation of $M_{1}$ in a unique way.

The discussion in the previous paragraph proves the following result.

Proposition 5.1.1. Let $\mathbf{G}$ be as in section 1.2 such that $\mathfrak{g}$ satisfies condition (3.1). Let $G$ be a finite topological central extension of $\mathbf{G}_{\mathbb{F}}$ represented as in (1.1). Let $P, L, N, \rho$ be as in the beginning of section 5.1. Then there exists a finite topological central extension $\tilde{G}$ of $\mathbf{G}_{\mathbb{F}}$ which covers $G$ as in

$$
1 \longrightarrow \tilde{F} \stackrel{\tilde{i}}{\longrightarrow} \tilde{G} \stackrel{\tilde{p}}{\longrightarrow} G \longrightarrow 1
$$

and a (closed) subgroup $P_{1}=M_{1} \cdot \tilde{N}$ of $\tilde{G}$ such that

i. $p \circ \tilde{p}\left(M_{1}\right)=\mathbf{M}_{\mathbb{F}}$ and $p \circ \tilde{p}(\tilde{N})=\mathbf{N}_{\mathbb{F}}$.

ii. $\tilde{N}$ is the unipotent radical of $\tilde{p}^{-1}(P)$; i.e. $\tilde{p}: \tilde{N} \mapsto N$ is an isomorphism.

iii. The representation $\rho$ (which can naturally be thought of as a representation of $\tilde{N}$ too) can be extended in a unique way to a representation of $P_{1}$.

iv. $M_{1}$ is perfect; i.e. $\left[M_{1}, M_{1}\right]=M_{1}$.

v. $P_{1}$ is a normal subgroup of $\tilde{p}^{-1}(P)$. 
5.2. Mackey analysis. In this section we assume that the notation is the same as in section 5.1. We also assume (without loss of generality) that

$$
\diamond \quad G \text { is equal to the extension } \tilde{G} \text { of Proposition [5.1.1 and }[G, G] \text { is dense in } G \text {. }
$$

Note that if $[G, G]$ is dense in $G$, then from [Tor, $\S 8.11]$ it follows that in fact $[G, G]=G$. The assumption $[G, G]=G$ is not a crucial one. This is because one can always find a closed finite-index subgroup $G_{1}$ of $G$ such that $G_{1}$ is perfect and $G=G_{1} \cdot \mathcal{Z}(G)$, and once a representation $\pi$ of $G$ is understood on $G_{1}$, It is easy to describe it as a representation of $G$.

Assume condition (5.1) holds. Let $P_{1} \subset G$ be the subgroup given in the statement of Proposition 5.1.1. Then we can write $P_{1}=M_{1} \cdot N$, where $M_{1}$ is defined in Proposition 5.1.1 and $N \approx \tilde{N}$ is the unipotent radical of the Heisenberg parabolic $P$ of $G$ which appears in parts ii and iii of Proposition 5.1.1.

Let $\pi$ be a unitary representation of $G$, without nonzero $G$-invariant vectors. Consider the restriction of $\pi$ to $P$. Recall that $\mathcal{Z}(N)$ means the center of $N$. By Howe-Moore theorem [HM], $\pi$ does not have a nonzero $\mathcal{Z}(N)$-invariant vector either. Therefore in the direct integral decomposition of $\pi$ as a representation of $N$, the spectral measure is supported on $\hat{N}_{\circ}$ (see the notation introduced at the end of section 2.2.).

Elements of $P_{1}$ commute with elements of $\mathcal{Z}(N)$. Therefore under the coadjoint action of $P$ on the unitary characters of $\mathcal{Z}(N), P_{1}$ lies within $\operatorname{stab}_{P}(\chi)$, the stabilizer (inside $P$ ) of any nontrivial additive unitary character $\chi$ of $\mathcal{Z}(N)$. In fact all these stabilizers are identical groups. We denote this common stabilizer group by $J$.

The action of $P$ on $\mathcal{Z}(N)$ (and also on its unitary characters) has only a finite number of orbits. Consequently, since $\pi$ has no nonzero $G$-invariant vectors, by elementary Mackey theory (see Theorems 3.11 and 3.12 of [Mac1] ) the restriction of $\pi$ to $P$ can be expressed as a finite direct sum

$$
\pi_{\mid P}=\bigoplus_{i} \operatorname{Ind}_{J}^{P} \sigma_{i}
$$

where each $\sigma_{i}$ is a representation of $J$ which, when restricted to $N$, is a direct integral of representations isomorphic to the representation $\rho_{i}$ of $N$ (defined in section 2.2) with some central character $\chi_{i}$. However, each $\rho_{i}$ extends in exactly one way to a representation of $P_{1}$. We still denote this extension by $\rho_{i}$. Again by Mackey theory we can write the restriction of $\sigma_{i}$ to $P_{1}$ as

$$
\sigma_{i \mid P_{1}}=\nu_{i} \otimes \rho_{i}
$$

where $\nu_{i}$ is indeed a representation of $M_{1}$ extended trivially on $N$ to $P_{1}$. Therefore we have proved the following result.

Lemma 5.2.1. Let $G, P, L$ and $N$ be as in the beginning of section 5.1] so that $G$ satisfies condition (5.1). Let $\pi$ be a unitary representation of $G$ without a nonzero $G$-invariant vector. Then $\pi$ can be written as a finite direct sum

$$
\pi_{\mid P}=\bigoplus_{i} \operatorname{Ind}_{J}^{P} \sigma_{i}
$$


where each $\sigma_{i}$ is a representation of $J$ such that

$$
\sigma_{i \mid P_{1}}=\nu_{i} \otimes \rho_{i}
$$

and $\nu_{i}$ factors to a representation of $M_{1}$. (In other words, $\nu_{i}$ is trivial on $N$.)

Recall the construction of the H-tower subgroup $U=N_{\Gamma}$ (see (3.4)). In the notation of (3.5) we have $H^{1}=N$. The quotient group $U_{2}=N_{\Gamma} / N$ is identical to the H-tower subgroup which is constructed for $M$. By Proposition 5.1.1, the latter H-tower group should lie within $M_{1}$.

Lemma 5.2.2. Let $\rho$ be the extension to $P_{1}$ of the irreducible representation of $N$ (which is also denoted by $\rho$ ) with an arbitrary nontrivial central character $\chi$ (see section 2.2). Let $N_{\Gamma}$ be as in (3.4). Then the restriction of the representation $\rho$ to $N_{\Gamma}$ is supported on rankable representations of $N_{\Gamma}$ of rank one.

Proof. The uniqueness of extension of $\rho$ to $P_{1}$ implies that on $M_{1}$, this extension should be identical to the extension obtained by restriction of extension of $\rho$ to the metaplectic group acting on $N / \mathcal{Z}(N)$. Therefore the lemma follows from Definition 4.1.1.

Notation. Let $K$ be an arbitrary (abstract) group and let $K^{\prime}$ be a subgroup of $K$. Let $\sigma$ be a representation of $K^{\prime}$ on a vector space $\mathcal{H}$. Let $a \in K$. Then by $\sigma^{a}$ we mean a representation of the subgroup $K_{a}^{\prime}=a K^{\prime} a^{-1}$ on $\mathcal{H}$ defined as follows:

$$
\text { for every } x \in K_{a}^{\prime}, \sigma^{a}(x)=\sigma\left(a^{-1} x a\right) \text {. }
$$

In particular, if $K^{\prime}=K$, then $\sigma^{a}$ is a representation of $K$.

At this point we recall Mackey's subgroup theorem. We will use it in sections 5.4 and 5.5. Giving a precise statement would require some definitions and would distract us from the main point of this paper. Therefore, for a detailed discussion we refer the reader to [Mac1, Theorem 3.5] or [Mac2, Theorem 12.1].

Theorem 5.2.3. (Mackey's Subgroup Theorem) Let $K$ be a locally compact group and let $K^{\prime}, K^{\prime \prime}$ be its closed subgroups. Assume that $K, K^{\prime}, K^{\prime \prime}$ are "nice" (see the above references for details). Let $\sigma$ be an irreducible representation of $K^{\prime}$. Then the representation

$$
\operatorname{Res}_{K^{\prime \prime}}^{K} \operatorname{Ind}_{K^{\prime}}^{K} \sigma
$$

has a direct integral decomposition supported on representations $\tau_{v}, v \in K$, where

$$
\tau_{v}=\operatorname{Ind}_{K^{\prime \prime} \cap K_{v}^{\prime}}^{K^{\prime \prime}} \operatorname{Res}_{K_{v}^{\prime \prime} \cap K_{v}^{\prime}}^{K^{\prime}} \sigma^{v} .
$$

Here

$$
K_{v}^{\prime}=v K^{\prime} v^{-1}
$$

and $\sigma^{v}$ is defined as in 5.4.

The following elementary lemma, which essentially follows from Mackey theory too, will help us in sections 5.4 and 5.5

Lemma 5.2.4. Let $K^{\prime} \subset K$ be two arbitrary locally compact groups such that $K^{\prime}$ is a closed normal subgroup of $K$. Let $\sigma$ be a unitary representation of $K$ which acts on the Hilbert space $\mathcal{H}$. Suppose

$$
\operatorname{Res}_{K^{\prime}}^{K} \sigma=\sigma_{1} \oplus \sigma_{2}
$$


where $\sigma_{1}$ and $\sigma_{2}$ are unitary representations of $K^{\prime}$ such that for any $a \in K$, we have

$$
\operatorname{Hom}_{K^{\prime}}\left(\sigma_{1}^{a}, \sigma_{2}\right)=\{0\} \text {. }
$$

(Here $\sigma_{1}^{a}$ is defined as in (5.4) and $\operatorname{Hom}_{K^{\prime}}\left(\sigma_{1}^{a}, \sigma_{2}\right)$ means the space of $K^{\prime}$-intertwining operators from $\sigma_{1}^{a}$ to $\left.\sigma_{2}.\right)$ For $i \in\{1,2\}$, let $\mathcal{H}_{i}$ be the closed $\sigma\left(K^{\prime}\right)$-invariant subspace of $\mathcal{H}$ which corresponds to the summand $\sigma_{i}$. Then each $\mathcal{H}_{i}$ is actually invariant under $\sigma(K)$.

Proof. Let $a \in K$. Then the linear operator $\mathcal{T}$ defined as

$$
\begin{aligned}
\mathcal{T}: \mathcal{H}_{1} & \mapsto \mathcal{H} \\
\mathcal{T}(w) & =\sigma(a) w \text { for all } w \in \mathcal{H}_{1}
\end{aligned}
$$

is an element of $\operatorname{Hom}_{K^{\prime}}\left(\sigma_{1}^{a}, \sigma\right)$. The orthogonal projection $\mathcal{P}$ of $\mathcal{H}$ onto $\mathcal{H}_{2}$ is an element of $\operatorname{Hom}_{K^{\prime}}\left(\sigma, \sigma_{2}\right)$. By the hypothesis of the lemma, one should have $\mathcal{P} \circ \mathcal{T}=0$. Therefore $\sigma(a) \mathcal{H}_{1} \subseteq \mathcal{H}_{1}$

5.3. Statement of the main theorems. We state the main theorems in this section and prove them in sections 5.4 and 5.5 .

Throughout this section, we assume $G, \mathbf{G}_{\mathbb{F}}$ and $\mathfrak{g}$ are as in section 1.2 and $\mathfrak{g}$ satisfies condition (3.1). Moreover, we assume $N_{\Gamma}$ is the H-tower subgroup of $G$ (see equality (3.4)), described as in (3.5).

Theorem 5.3.1. Let $\pi$ be a unitary representation of $G$. Then in the direct integral decomposition

$$
\pi_{\mid N_{\Gamma}}=\int_{\hat{N}_{\Gamma}}^{\oplus} \tau d \mu(\tau)
$$

the support of the spectral measure $\mu$ is inside the subset of rankable representations of $N_{\Gamma}$.

In the next theorem the subset of rankable representations of rank $k$ of $N_{\Gamma}$ will be denoted by $\hat{N}_{\Gamma}(k)$.

Theorem 5.3.2. Let $\pi$ be a unitary representation of $G$ on a Hilbert space $\mathcal{H}_{\pi}$. Consider the direct integral decomposition

$$
\pi_{\mid N_{\Gamma}}=\int_{\hat{N}_{\Gamma}}^{\oplus} \tau d \mu(\tau)
$$

and let $\mathcal{P}_{\mu}$ be the projective measure corresponding to this decomposition. Let $\hat{N}_{\Gamma}(\pi)$ be the support of $\pi$ in this direct integral decomposition. Set

$$
\mathcal{H}_{\pi}^{j}=\mathcal{P}_{\mu}\left(\hat{N}_{\Gamma}(j) \cap \hat{N}_{\Gamma}(\pi)\right) \cdot \mathcal{H}_{\pi} \quad \text { for any } 0 \leq j \leq \operatorname{ht}\left(N_{\Gamma}\right) .
$$

Then for every $j$ such that

$$
j \in\left\{0,1,2, \ldots, \operatorname{ht}\left(N_{\Gamma}\right)\right\}
$$

$\mathcal{H}_{\pi}^{j}$ is a $G$-invariant subspace of $\mathcal{H}_{\pi}$. The direct sum of all these subspaces is equal to $\mathcal{H}_{\pi}$. 
Remark. It is clear that for $\mathcal{P}_{\mu}\left(\hat{N}_{\Gamma}(j) \cap \hat{N}_{\Gamma}(\pi)\right)$ to make sense we need to show that the sets $\hat{N}_{\Gamma}(j) \cap \hat{N}_{\Gamma}(\pi)$ are indeed Borel subsets of $\hat{N}_{\Gamma}$. In fact one can show that the set of rankable representations of a given rank of an H-tower group $U=H^{1} \cdots H^{t}$ can be constructed with a finite number of set-theoretic operations on open and closed sets of the unitary dual of $U$. Here is a sketch of the proof. Any rankable representation $\sigma$ is a tensor product of the form

$$
\rho_{1} \otimes \cdots \otimes \rho_{t}
$$

such that $\rho_{1}, \ldots, \rho_{\operatorname{rank}(\sigma)}$ are extensions of representations (with nontrivial central characters) of $H^{1}, \ldots, H^{\operatorname{rank}(\sigma)}$ respectively, and the rest of the $\rho_{j}$ 's are trivial. The first requirement imposes open conditions on the subset of rankable representations of a given rank, whereas the second requirement imposes a closed condition.

Definition 5.3.3. Let $\pi$ be any unitary representation of $G$. $\pi$ is called "pure-rank" if its restriction to $N_{\Gamma}$ is a direct integral of rankable representations of a fixed rank. The common rank of these rankable representations is called the rank of $\pi$.

Although Theorem 5.3.2 is slightly stronger than Corollary 5.3.4 below, we would like to state it in order to clarify the analogy between our new theory and the older one.

Corollary 5.3.4. Let $\pi$ be an irreducible representation of $G$. Then $\pi$ is pure-rank.

5.4. Proof of Theorem [5.3.1. In this section we prove Theorem 5.3.1. Without loss of generality we can assume that condition (5.1) holds. Theorem 5.3.1 is proved by induction on $h t\left(N_{\Gamma}\right)$. Let $N_{\Gamma}$ be described as in (3.5). By Howe-Moore's theorem [HM], when $\operatorname{ht}\left(N_{\Gamma}\right)=1$ there is nothing to prove. Now let $\operatorname{ht}\left(N_{\Gamma}\right)>1$. Let $P, L, M$ and $N$ be as in section [5.1. Let $P_{1}, M_{1}$ be as in Proposition 5.1.1. The H-tower subgroup which is associated to $M$ is $N_{2}=N_{\Gamma} / N$ and it lies within $M_{1}$. But $\operatorname{ht}\left(N_{2}\right)=\operatorname{ht}\left(N_{\Gamma}\right)-1$, and therefore Theorem 5.3.1 holds for $M_{1}$.

Without loss of generality, we can assume $\pi$ has no nonzero $G$-fixed vectors. Let $\sigma_{i}$ 's be the representations which appear in the decomposition of $\pi$ using Lemma 5.2.1. By a straightforward application of Mackey's subgroup theorem (see Theorem 5.2.3), or even more directly (with only little difficulty) by writing the definition of the induced representation $\operatorname{Ind}_{J}^{P} \sigma_{i}$ explicitly, one can see that the representation

$$
\operatorname{Res}_{P_{1}}^{P} \operatorname{Ind}_{J}^{P} \sigma_{i}
$$

is supported on representations of the form

$$
\operatorname{Res}_{P_{1}}^{J} \sigma_{i}^{x} \quad x \in P
$$

where $\sigma_{i}^{x}$ is defined as in (5.4); i.e.

$$
\text { for any } y \in J, \sigma_{i}^{x}(y)=\sigma_{i}\left(x^{-1} y x\right) \text {. }
$$

Note that $J$ is a normal subgroup of $P$ because it contains $[P, P]$.

By (5.3), $\operatorname{Res}_{P_{1}}^{J} \sigma_{i}^{x}$ is unitarily equivalent to $\nu_{i}^{x} \otimes \rho_{i}^{x}$ where $\nu_{i}^{x}$ and $\rho_{i}^{x}$ are defined similar to (5.5). Theorem 5.3.1 follows from Lemma 5.2.2 applied to $\rho_{i}^{x}$, the fact that by induction hypothesis $\nu_{i}^{x}$ is supported on rankable representations of $N_{2}=N_{\Gamma} / N$, and Definition 4.1 .1 
5.5. Proof of Theorem 5.3.2. We will now proceed towards proving Theorem 5.3.2. Without loss of generality, we can assume that condition (5.1) holds. Let $P, L, N$ and $M$ be as in section [5.1. Let $P_{1}, M_{1}$ be as in Proposition 5.1.1. The main idea behind the proof is that the Kirillov orbits of representations of different rank have different dimensions. We will apply some basic Kirillov theory.

Recall from section 5.3 that $G, \mathfrak{g}, \mathfrak{g}_{\mathbb{F}}$ are as in section 1.2 and $\mathfrak{g}$ satisfies condition (3.1). Let $\Sigma, \Sigma^{+}$and $\Sigma_{B}$ be as in section 1.2 too. Let $\tilde{\beta}$ be the highest root of $\mathfrak{g}$ (see section 1.2) and $\beta$ be a simple restricted root such that $(\beta, \tilde{\beta})=1$. Recall that

$$
P_{\{\beta\}}=L_{\{\beta\}} N_{\{\beta\}}
$$

is a standard parabolic subgroup of $G$ associated to $\{\beta\}$ (see section [1.2). Let $N_{\Gamma}$ be the H-tower subgroup of $G$ and suppose $\mathfrak{n}_{\Gamma}$ is its Lie algebra. Let $N_{\{\beta\}}$ be as in equation (5.6) and suppose $\mathfrak{n}_{\{\beta\}}$ is its Lie algebra. We consider $\mathfrak{n}_{\Gamma}$ and $\mathfrak{n}_{\{\beta\}}$ as subalgebras of $\mathfrak{g}_{\mathbb{F}}$. Recall that for any $\gamma \in \Sigma,\left(\mathfrak{g}_{\mathbb{F}}\right)_{\gamma}$ denotes the restricted root subspace of $\mathfrak{g}_{\mathbb{F}}$ associated to $\gamma$. Our next aim is to define a subgroup $N_{\Gamma}^{\beta}$ of $N_{\Gamma}$ in case $\operatorname{ht}\left(N_{\Gamma}\right)>1$.

Let $L_{\Gamma}$ be as in equation (3.4), with Lie algebra $\mathfrak{l}_{\Gamma}$ where $\mathfrak{l}_{\Gamma} \subset \mathfrak{g}_{\mathbb{F}}$, and

$$
\Sigma^{L}=\left\{\gamma \in \Sigma \mid\left(\mathfrak{g}_{\mathbb{F}}\right)_{\gamma} \subset \mathfrak{l}_{\Gamma}\right\} .
$$

Define

$$
\mathfrak{n}_{\Gamma}^{\beta}=\bigoplus_{\gamma \in S_{\Gamma}}\left(\mathfrak{g}_{\mathbb{F}}\right)_{\gamma}
$$

where

$$
S_{\Gamma}=\left\{\gamma \in \Sigma^{+} \mid\left(\mathfrak{g}_{\mathbb{F}}\right)_{\gamma} \subset \mathfrak{n}_{\Gamma} \cap \mathfrak{n}_{\{\beta\}} \text { and } \gamma-\beta \notin \Sigma^{L}\right\} .
$$

One can see that $\mathfrak{n}_{\Gamma}^{\beta}$ is a Lie subalgebra of $\mathfrak{g}_{\mathbb{F}}$. To see this, assume $\gamma, \gamma^{\prime} \in S_{\Gamma}$ and $\gamma+\gamma^{\prime} \in \Sigma^{+}$. Then since both $\mathfrak{n}_{\Gamma}$ and $\mathfrak{n}_{\{\beta\}}$ are Lie algebras, $\left(\mathfrak{g}_{\mathbb{F}}\right)_{\gamma+\gamma^{\prime}} \subset \mathfrak{n}_{\Gamma} \cap \mathfrak{n}_{\{\beta\}}$. Moreover, if $\gamma+\gamma^{\prime}-\beta \in \Sigma^{L}$, then either $\gamma \in \Sigma^{L}$ or $\gamma^{\prime} \in \Sigma^{L}$ which is a contradiction. Consequently $\gamma+\gamma^{\prime} \in S_{\Gamma}$.

The group $N_{\Gamma}^{\beta}$ is defined as the subgroup of $N_{\Gamma} \cap N_{\{\beta\}}$ with Lie algebra $\mathfrak{n}_{\Gamma}^{\beta}$. (It is worth mentioning that $N_{\Gamma}^{\beta}$ is a proper subgroup of $N_{\Gamma} \cap N_{\{\beta\}}$ if and only if $\Gamma$ contains an element which is not orthogonal to $\beta$ in $\Sigma$.)

Lemma 5.5.1. Let $\mathrm{ht}\left(N_{\Gamma}\right)>1$ and $L_{\{\beta\}}$ be as in equation (5.6). Let $M_{\{\beta\}}=\left[L_{\{\beta\}}, L_{\{\beta\}}\right]$, the commutator subgroup of $L_{\{\beta\}}$. Then $M_{\{\beta\}}$ normalizes $N_{\Gamma}^{\beta}$.

Proof. It suffices to show that for any $\gamma \in S_{\Gamma}$, if $\gamma+\beta \in \Sigma$ (respectively $\gamma-\beta \in \Sigma$ ) then $\gamma+\beta \in S_{\Gamma}$ (respectively $\gamma-\beta \in S_{\Gamma}$ ). Note that $\gamma-\beta$ cannot be zero.

Assume $\gamma \in S_{\Gamma}$ and $\gamma+\beta \in \Sigma$. Since $\left(\mathfrak{g}_{\mathbb{F}}\right)_{\gamma} \subset \mathfrak{n}_{\{\beta\}}$, we have $\left(\mathfrak{g}_{\mathbb{F}}\right)_{\gamma+\beta} \subset \mathfrak{n}_{\{\beta\}}$ too. Similarly, since $\left(\mathfrak{g}_{\mathbb{F}}\right)_{\gamma} \subset \mathfrak{n}_{\Gamma}, \gamma+\beta \notin \Sigma^{L}$ and $(\gamma+\beta)-\beta=\gamma \notin \Sigma^{L}$ which imply that $\gamma+\beta \in S_{\Gamma}$.

Next assume $\gamma \in S_{\Gamma}$ and $\gamma-\beta \in \Sigma$. Note that $\gamma-\beta \in \Sigma$ implies $\gamma-\beta \in \Sigma^{+}$since $\gamma \in \Sigma^{+}$. Again since $\left(\mathfrak{g}_{\mathbb{F}}\right)_{\gamma} \subset \mathfrak{n}_{\{\beta\}}$, we have $\left(\mathfrak{g}_{\mathbb{F}}\right)_{\gamma-\beta} \in \mathfrak{n}_{\{\beta\}}$. Moreover, by the definition of 
$\mathfrak{n}_{\Gamma}^{\beta}, \gamma-\beta \notin \Sigma^{L}$ and therefore $\left(\mathfrak{g}_{\mathbb{F}}\right)_{\gamma-\beta} \subset \mathfrak{n}_{\Gamma}$. Finally, if $(\gamma-\beta)-\beta \in \Sigma^{L}$, then $\gamma=2 \beta+\gamma_{1}$ where $\gamma_{1} \in \Sigma^{L}$. This means that $(\gamma, \tilde{\beta})=2$, i.e. $\gamma$ is the highest root. Since any simple restricted root appears in the highest root with a positive coefficient, it follows that $\Sigma_{B}$ consists of $\beta$ and the elements of $\Gamma$. Consequently, there is only one simple restricted root outside $\Gamma$, which implies that $\operatorname{ht}\left(N_{\Gamma}\right)=1$, which contradicts our assumption.

Proposition 5.5.2. Let $G$ be as in Theoreom 5.3.2, $N_{\Gamma}$ be the H-tower subgroup of $G$ and $\operatorname{ht}\left(N_{\Gamma}\right)>1$. Let $N_{\Gamma}^{\beta}$ be defined as in equation (5.7). Then the restriction of a rankable representation $\sigma$ of rank $k$ of $N_{\Gamma}$ to $N_{\Gamma}^{\beta}$ is a direct integral of irreducible representations of $N_{\Gamma}^{\beta}$ whose attached coadjoint orbits have the same dimension equal to $2\left(n_{1}+\cdots+n_{k}-c\right)$, where $c$ is the codimension of $N_{\Gamma}^{\beta}$ in $N_{\Gamma}$, and $n_{i}$ 's are defined as in Corollary 4.2.3.

Proof. Throughout the proof we assume $\mathbb{F}=\mathbb{R}$ for simplicity. The proof for other local fields is essentially similar.

We will analyze

$$
\operatorname{Res}_{N_{\Gamma}^{\beta}}^{N_{\Gamma}}\left(\rho_{1} \otimes \cdots \otimes \rho_{k}\right)
$$

with the $\rho_{i}$ 's as in Definition 4.1.1. It is easy to see that $N_{\Gamma}^{\beta} \supset H^{2} \cdots H^{\text {ht }\left(N_{\Gamma}\right)}$, so when $j>1$ the restriction of $\rho_{j}$ to $N_{\Gamma}^{\beta}$ is irreducible. It remains to understand the restriction of $\rho_{1}$ to $N_{\Gamma}^{\beta}$. The group $H^{1} \cap N_{\Gamma}^{\beta}$ is a direct product of a Heisenberg group of dimension $2\left(n_{1}-c\right)+1$ and a $c$-dimensional abelian group whose Lie algebra corresponds to the direct sum of restricted root spaces $\left(\mathfrak{g}_{\mathbb{R}}\right)_{\tilde{\beta}-\gamma}$, such that $(\gamma, \tilde{\beta})>0$ but $\gamma \notin S_{\Gamma}$. To see why these restricted root spaces form an isotropic subspace of the Heisenberg nilradical of $\mathfrak{g}_{\mathbb{R}}$, suppose $\tilde{\beta}-\gamma_{1}, \tilde{\beta}-\gamma_{2}$ are given such that for any $i \in\{1,2\},\left(\gamma_{i}, \tilde{\beta}\right)>0$ but $\gamma_{i} \notin S_{\Gamma}$. If $\left(\tilde{\beta}-\gamma_{1}\right)+\left(\tilde{\beta}-\gamma_{2}\right)=\tilde{\beta}$, then $\tilde{\beta}=\gamma_{1}+\gamma_{2}$. But for any $i \in\{1,2\}, \gamma_{i}=\beta+\gamma_{i}^{\prime}$ where $\gamma_{i}^{\prime} \in \Sigma^{L}$ or $\gamma_{i}^{\prime}=0$. Therefore $\tilde{\beta}=2 \beta+\gamma_{1}^{\prime}+\gamma_{2}^{\prime}$, which implies that $\Sigma_{B}$ consists of $\beta$ and the elements of $\Gamma$. (See the proof of Lemma 5.5.1.) Consequently $\operatorname{ht}\left(N_{\Gamma}\right)=1$, which is a contradiction.

Let the decomposition of $H^{1} \cap N_{\Gamma}^{\beta}$ as a direct product be $H^{\beta} \times \mathbb{R}^{c}$, where $H^{\beta}$ is the $2\left(n_{1}-c\right)+1$-dimensional Heisenberg group and $\mathbb{R}^{c}$ is the abelian factor. We will denote the irreducible representation of $H^{\beta}$ with central character $\chi_{1}$ by $\rho_{1}^{\beta}$.

Lemma 5.5.3. Under the foregoing assumptions,

$$
\operatorname{Res}_{H^{1} \cap N_{\Gamma}^{\beta}}^{N_{\Gamma}} \rho_{1}=\int_{\mathbb{R}^{c *}} \rho_{1}^{\beta} \hat{\otimes} \psi_{s} d \mu(s)
$$

where each $\psi_{s}(t)$, given by $\psi_{s}(t)=e^{\mathrm{i} s(t)}$ for some $s \in \mathbb{R}^{c *}$, the vector-space dual of $\mathbb{R}^{c}$, is a unitary character of $\mathbb{R}^{c}$.

Proof. Clearly

$$
\operatorname{Res}_{H^{1} \cap N_{\Gamma}^{\beta}}^{N_{\Gamma}} \rho_{1}=\operatorname{Res}_{H^{\beta} \times \mathbb{R}^{c}}^{H^{1}} \operatorname{Res}_{H^{1}}^{N_{\Gamma}} \rho_{1}=\operatorname{Res}_{H^{\beta} \times \mathbb{R}^{c}}^{H^{1}} \rho_{1}
$$


The space $\mathcal{H}_{\rho}$ of any representation $\rho$ of the Heisenberg group $H^{1}=H_{n_{1}}$ introduced in section 2.2 can be written as

$$
\begin{aligned}
\mathcal{H}_{\rho}=L^{2}(\mathfrak{y})= & L^{2}\left(\operatorname{Span}_{\mathbb{R}}\left\{Y_{1}, \ldots, Y_{c}\right\} \oplus \operatorname{Span}_{\mathbb{R}}\left\{Y_{c+1}, \ldots, Y_{n_{1}}\right\}\right)= \\
& L^{2}\left(\mathbb{R}^{c}\right) \hat{\otimes} L^{2}\left(\operatorname{Span}_{\mathbb{R}}\left\{Y_{c+1}, \ldots, Y_{n_{1}}\right\}\right) \approx \int_{\mathbb{R}^{c *}}^{\oplus} L_{s}^{2} d \mu(s)
\end{aligned}
$$

where each $L_{s}^{2}$ is equal to $L^{2}\left(\operatorname{Span}_{\mathbb{R}}\left\{Y_{c+1}, \ldots, Y_{n_{1}}\right\}\right)$ on which $\mathbb{R}^{c}$ acts via the character $\chi_{s}(x)=e^{\mathrm{i} s(x)}$. In fact $L_{s}^{2}$ is a representation of $H^{\beta} \times \mathbb{R}^{c}$. Lemma 5.5.3 is proved.

Next we show that $\mathbb{R}^{c} \subset \mathcal{Z}\left(N_{\Gamma}^{\beta}\right)$. To see this, take a restricted root space $\left(\mathfrak{g}_{\mathbb{R}}\right)_{\tilde{\beta}-\gamma}$ which lies inside $\mathbb{R}^{c}$. It suffices to show that for any $\gamma^{\prime} \in S_{\Gamma},(\tilde{\beta}-\gamma)+\gamma^{\prime} \notin \Sigma$. But we know that $\gamma=\beta+\gamma_{1}$ where $\gamma_{1} \in \Sigma^{L}$ or $\gamma_{1}=0$. Therefore $(\tilde{\beta}-\gamma)+\gamma^{\prime}=\tilde{\beta}-\beta-\gamma_{1}+\gamma^{\prime}$. Consequently, if $(\tilde{\beta}-\gamma)+\gamma^{\prime} \in \Sigma$, then either $\gamma^{\prime} \in \Sigma^{L}$ or $\gamma^{\prime}-\beta=\gamma_{1} \in \Sigma^{L}$. However, none of these is possible for $\gamma^{\prime}$ by the definition of $S_{\Gamma}$ in (5.8).

We have shown that $\mathbb{R}^{c} \subset \mathcal{Z}\left(N_{\Gamma}^{\beta}\right)$ and the action of $\mathbb{R}^{c}$ on each $L_{s}^{2}$ is by a distinct character $\psi_{s}$ (see Lemma 5.5.3). Hence the restriction of $\rho_{1}$ to $N_{\Gamma}^{\beta}$ breaks into a direct integral of a $c$-parameter family of irreducible representations. Consequently the same thing happens to any rankable representation

$$
\sigma=\rho_{1} \otimes \cdots \otimes \rho_{k}
$$

By Theorem 2.5.1 of [CG] and Lemma 4.2.2 applied to $N_{\Gamma}^{\beta}$ which is a semidirect product of $H^{\beta} \times \mathbb{R}^{c}$ and $N_{2}$, it follows that the projection of the coadjoint orbit $\mathcal{O}_{\sigma}^{*}$ onto the Lie algebra of $N_{\Gamma}^{\beta}$ is foliated by subvarieties of codimension $2 c$, which are indeed coadjoint orbits of the constituents of the rankable representations in the restriction of $\sigma$ to $N_{\Gamma}^{\beta}$. Proposition 5.5 .2 is proved.

We will now concentrate on finishing the proof of Theorem 5.3 .2 Theorem 5.3 .2 is proved by induction on the height $\mathrm{ht}\left(N_{\Gamma}\right)$ of the H-tower group $N_{\Gamma}$. If $\operatorname{ht}\left(N_{\Gamma}\right)=1$ then there is nothing to prove. Let $\operatorname{ht}\left(N_{\Gamma}\right)>1$. Then the H-tower subgroup of $M_{1}$ is equal to $N_{2}$, where

Clearly ht $\left(N_{2}\right)=\operatorname{ht}\left(N_{\Gamma}\right)-1$.

$$
N_{2}=N_{\Gamma} / N
$$

Without loss of generality we can assume $\pi$ has no nonzero $G$-invariant vectors. Consider the decomposition of $\pi$ given in (5.2). Applying induction hypothesis to $M_{1}$, which contains the H-tower subgroup $N_{2}$, we can refine this decomposition by expressing each $\nu_{i}$ as a direct sum of its $M_{1}$-invariant pure-rank parts (where the rank for a representation of $M_{1}$ is naturally defined with respect to $N_{2}$ ). Conseqently, as a representation of $M_{1}$,

$$
\nu_{i}=\nu_{i, 0} \oplus \nu_{i, 1} \oplus \cdots \oplus \nu_{i, \mathrm{ht}\left(N_{2}\right)}
$$

Here $\nu_{i, j}$ denotes the part of $\nu_{i}$ supported on rankable representations of $N_{2}$ of rank $j$. (Note that some of the $\nu_{i, j}$ 's may be trivial, but this fact does not affect our proof.) Therefore we have

$$
\sigma_{i \mid P_{1}}=\left(\nu_{i, 0} \otimes \rho_{i}\right) \oplus \cdots \oplus\left(\nu_{i, \mathrm{ht}\left(N_{2}\right)} \otimes \rho_{i}\right) .
$$

Let $\mathcal{H}$ be the Hilbert space of the representation $\sigma_{i}$. For any $j \in\left\{0,1,2, \ldots, \operatorname{ht}\left(N_{2}\right)\right\}$, let $\mathcal{H}_{j}$ be the subspace of $\mathcal{H}$ which corresponds to $\nu_{i, j} \otimes \rho_{i}$. Our next task is to prove that 
each $\mathcal{H}_{j}$ is in fact invariant under $\sigma_{i}(J)$. (See Lemma 5.2.1) This follows from Lemma 5.2 .4 once we prove the following lemma.

Lemma 5.5.4. Let $\eta=\nu_{i, j} \otimes \rho_{i}$ and $\eta^{\prime}=\nu_{i, j^{\prime}} \otimes \rho_{i}$ where $j \neq j^{\prime}$. Let $a \in J$. Then

$$
\operatorname{Hom}_{P_{1}}\left(\eta^{a}, \eta^{\prime}\right)=\{0\}
$$

where $\eta^{a}$ is a representation of $P_{1}$ defined on $\mathcal{H}_{j}$ defined as in (5.4); i.e.

$$
\eta^{a}(x)=\eta\left(a^{-1} x a\right) \quad \text { for all } x \in P_{1} .
$$

Proof. We actually prove more; i.e. that

$$
\operatorname{Hom}_{N_{\Gamma}}\left(\operatorname{Res}_{N_{\Gamma}}^{P_{1}} \eta^{a}, \operatorname{Res}_{N_{\Gamma}}^{P_{1}} \eta^{\prime}\right)=\{0\}
$$

We claim that $\operatorname{Res}_{N_{\Gamma}}^{P_{1}} \eta^{a}$ is a direct integral supported on rankable representations of $N_{\Gamma}$ of rank $j+1$. The claim implies (5.9) because if $j \neq j^{\prime}$, then it implies that $\operatorname{Res}_{N_{\Gamma}}^{P_{1}} \eta^{a}$ and $\operatorname{Res}_{N_{\Gamma}}^{P_{1}} \eta^{\prime}$ are supported on disjoint subsets of $\hat{N}_{\Gamma}$. We prove this claim below.

By Lemma 5.2.2, $\rho_{i}^{a}$ is supported on rankable representations of $N_{\Gamma}$ of rank one. Next we show that $\nu_{i, j}^{a}$ is supported on rankable representations of $N_{2}=N_{\Gamma} / N$ of rank $j$.

As we know, $G$ is a central extension of $\mathbf{G}_{\mathbb{F}}$. Suppose this extension is represented as in (1.1).

First let $a \in N$. Then $a \in N_{\Gamma}$ and consequently $\operatorname{Res}_{N_{\Gamma}}^{P_{1}} \eta^{a}$ is unitarily equivalent to $\operatorname{Res}_{N_{\Gamma}}^{P_{1}} \eta$. Since $J \subset P$ and any element of $P$ is a product of an element of $N$ and an element of $p^{-1}\left(\mathbf{L}_{\mathbb{F}}\right)$, it follows that it suffices to assume that $p(a) \in \mathbf{L}_{\mathbb{F}}$.

It follows from $p(a) \in \mathbf{L}_{\mathbb{F}}$ that $p(a) \mathbf{M}_{\mathbb{F}} p\left(a^{-1}\right)=\mathbf{M}_{\mathbb{F}}$. The group $p\left(P_{\Gamma}\right)$ is the $\mathbb{F}$-points of the $\mathbb{F}$-parabolic $\mathbf{P}_{\boldsymbol{\Gamma}^{\prime}}$ of $\mathbf{G}$. Now $\mathbf{P}_{\mathbf{m}}=\mathbf{P}_{\boldsymbol{\Gamma}^{\prime}} \cap \mathbf{M}$ is an $\mathbb{F}$-parabolic of $\mathbf{M}$. Since $p(a) \in \mathbf{L}_{\mathbb{F}}, p(a) \mathbf{P}_{\mathbf{m}} p\left(a^{-1}\right)$ is another $\mathbb{F}$-parabolic of $\mathbf{M}$, and therefore by Bor, Theorem $20.9]$ these two parabolics are conjugate under an element $p(b)$ of $\mathbf{M}_{\mathbb{F}}$ where $b \in M_{1}$ (recall from Proposition 5.1.1 that $\left.p\left(M_{1}\right) \supseteq \mathbf{M}_{\mathbb{F}}\right)$. This means that $p(b a) \mathbf{P}_{\mathbf{m}} p(b a)^{-1}=\mathbf{P}_{\mathbf{m}}$. If $\mathbf{U}_{\mathbf{m}}$ is the unipotent radical of $\mathbf{P}_{\mathbf{m}}$, then $p(b a) \mathbf{U}_{\mathbf{m}} p(b a)^{-1}=\mathbf{U}_{\mathbf{m}}$, which implies that $b_{a} N_{2} a^{-1} b^{-1}=N_{2}$.

Let $c=b a$. Then $c \in P$. Let $\mathfrak{n}_{2} \subset \mathfrak{n}_{\Gamma}$ be the Lie algebra of $N_{2}$. Consider $A d^{*}(c)$ as a linear map from the dual of the Lie algebra of $P$ to itself. Obviously $A d^{*}(c)\left(\mathfrak{n}_{2}^{*}\right)=\mathfrak{n}_{2}^{*}$. Let $\tau$ be an irreducible unitary representation of $N_{2}$ and let $\mathcal{O}_{\tau}^{*}$ be the coadjoint orbit associated to $\tau$. Then the coadjoint orbit associated to $\tau^{c}$ is $A d^{*}(c)\left(\mathcal{O}_{\tau}^{*}\right)$. This fact follows for instance from [Du, III 11]. A short proof of this fact can be obtained by an adaptation of the proof of Lemma 5.5.6.

For simplicity let $\nu=\nu_{i, j}$ for fixed $i, j$. The representation $\operatorname{Res}_{N_{2}}^{M_{1}} \nu$ is supported on rankable representations of $N_{2}$ whose associated coadjoint orbits have dimension

$$
n_{2}+\cdots+n_{j+1}
$$

(See Corollary 4.2.3 for the definition of $n_{i}$ 's.) 
Let $\nu^{a}, \nu^{b}, \nu^{c}$ be representations of $M_{1}$ defined as in (5.4). (Note that $a M_{1} a^{-1}=M_{1}$ because $P_{1}$ is normal in $p^{-1}\left(\mathbf{P}_{\mathbb{F}}\right)$.) Since $b \in M_{1}$, any representation $\theta$ of $M_{1}$ is obviously unitarily equivalent to $\theta^{b}$. Therefore $\nu^{c}$ is unitarily equivalent to $\nu^{a}$. Since the action of $A d^{*}(c)$ on $\mathfrak{n}_{2}^{*}$ is linear, it does not change the dimension of coadjoint orbits, and therefore $\operatorname{Res}_{N_{2}}^{M_{1}} \nu^{c}$ is supported on unitary representations of $N_{2}$ whose associated coadjoint orbits have dimension $n_{2}+\cdots+n_{j+1}$.

Since $\nu^{c}$ is a unitary representation of $M_{1}$, by Theorem 5.3.1 all representations in the support of its restriction to $N_{2}$ should be rankable. Therefore $\operatorname{Res}_{N_{2}}^{M_{1}} \nu^{c}$ is supported on rankable representations of $N_{2}$ of rank $j$. Now $\nu^{a}$ is unitarily equivalent to $\nu^{c}$ as a representation of $M_{1}$, and hence as a representation of $N_{2}$. Therefore $\nu^{a}$ is also supported on rankable representations of $N_{2}$ of rank $j$. Definition 4.1.1 completes the proof of our claim. The proof of Lemma 5.5 .4 is complete.

As mentioned before, Lemma 5.5.4 implies that each of the subspaces $\mathcal{H}_{j}$ (which corresponds to the representation $\nu_{i, j} \otimes \rho_{i}$ of $\left.P_{1}\right)$ is invariant under $\sigma_{i}(J)$.

Lemma 5.5.5. There is a P-invariant direct sum decomposition of $\pi$ such as

$$
\pi_{\mid P}=\pi_{1} \oplus \cdots \oplus \pi_{\mathrm{ht}\left(N_{\Gamma}\right)}
$$

where each $\pi_{i}$ is of pure $N_{\Gamma}$-rank $i$.

Proof. The invariance of the spaces $\mathcal{H}_{j}$ under $\sigma_{i}(J)$ means that one can actually decompose $\sigma_{i}$ as a direct sum

$$
\sigma_{i}=\sigma_{i, 1} \oplus \sigma_{i, 2} \oplus \cdots \oplus \sigma_{i, \mathrm{ht}\left(N_{\Gamma}\right)}
$$

such that each $\sigma_{i, j}$ is a representation of $J$ and

$$
\sigma_{i, j \mid P_{1}}=\nu_{i, j-1} \otimes \rho_{i} .
$$

Now an application of Mackey's subgroup theorem (see Theorem [5.2.3) immediately implies that the representation

$$
\operatorname{Res}_{N_{\Gamma}}^{P} \operatorname{Ind}_{J}^{P} \sigma_{i, j}
$$

is supported on rankable representations of $N_{\Gamma}$ of rank $j$. (See the argument of Theorem 5.3.1 and Lemma 5.5.4) Lemma 5.5.5 is proved.

Recall from Lemma 5.5.1 that the group $N_{\Gamma}^{\beta}$ is normalized by $M_{\{\beta\}}$. A version of the following lemma was mentioned in the proof of Lemma 5.5.4.

Lemma 5.5.6. Let $\tau$ be a unitary representation of $N_{\Gamma}^{\beta}$ and let $g \in M_{\{\beta\}}$. Let $\tau^{g}$ be defined as in (5.4). Then the coadjoint orbits associated to $\tau$ and $\tau^{g}$ (in the sense of Kirillov's orbital theory) have the same dimension.

Proof. The proof follows immediately from the fact that the coadjoint orbit attached to $\tau^{g}$ is $A d^{*}(g)\left(\mathcal{O}^{*}\right)$, which follows from [Du, III 11]. We would like to give a short proof of this fact for the reader's convenience. Let $\mathfrak{n}_{\Gamma}^{\beta}$ be the Lie algebra of $N_{\Gamma}^{\beta}$. Fix an additive character $\chi$ of $\mathbb{F}$ as done in $[\mathrm{Mo1}, \S 4]$. (When $\mathbb{F}=\mathbb{R}$ or $\mathbb{C}, \chi(t)=e^{\mathrm{iRe}(t)}$ where $\operatorname{Re}(t)$ means the real part of $t$, and when $\mathbb{F}$ is $p$-adic, $\chi$ will be an unramified character given by Tate.) Let the coajoint orbit associated to $\tau$ be $\mathcal{O}_{\tau}^{*} \subset \mathfrak{n}_{\Gamma}^{\beta}$. By Kirillov's orbital theory we know that $\tau$ is constructed as follows. One chooses an arbitrary element $\lambda \in \mathcal{O}_{\tau}^{*}$ and a 
maximal subalgebra $\mathfrak{q}$ of $\mathfrak{n}_{\Gamma}^{\beta}$ subordinate to $\lambda$, which exponentiates to a closed subgroup $Q$ of $N_{\Gamma}^{\beta}$. Then

$$
\tau=\operatorname{Ind}_{Q}^{N_{\Gamma}^{\beta}}(\chi \circ \lambda \circ \log ) .
$$

For any $g \in M_{\{\beta\}}$, let $(\chi \circ \lambda \circ \log )^{g}$ be defined as in (5.4). Then

$$
\tau^{g}=\operatorname{Ind}_{g Q g^{-1}}^{N_{\Gamma}^{\beta}}(\chi \circ \lambda \circ \log )^{g}=\operatorname{Ind}_{g Q g^{-1}}^{N_{\Gamma}^{\beta}}\left(\chi \circ A d^{*}(g)(\lambda) \circ \log \right) .
$$

Since $A d(g)(\mathfrak{q})$ is a maximal subalgebra subordinate to $A d^{*}(g)(\lambda)$, the coadjoint orbit attached to $\tau^{g}$ is

$$
\operatorname{Ad}^{*}(g)\left(\mathcal{O}^{*}\right) \text {. }
$$

Since the action of $A d^{*}(g)$ is linear on $\mathfrak{n}_{\Gamma}^{\beta}$, it does not change the dimension of the coadjoint orbit.

To prove Theorem 5.3.2, we show that each of the components $\pi_{i}$ given in Lemma 5.5.5 is $G$-invariant as well. To this end, we first prove the following lemma.

Lemma 5.5.7. The P-invariant decomposition in Lemma5.5.5 is preserved by the action of $M_{\{\beta\}}$, where

$$
M_{\{\beta\}}=\left[L_{\{\beta\}}, L_{\{\beta\}}\right]
$$

and $L_{\{\beta\}}$ is the Levi component of the standard parabolic $P_{\{\beta\}}$ with $\beta$ as in Proposition 5.5.2.

Proof. For any $1 \leq j \leq h t\left(N_{\Gamma}\right)$, the representation

$$
\operatorname{Res}_{N_{\Gamma}^{\beta}}^{P} \pi_{j}
$$

is a direct integral of representations which correspond to coadjoint orbits of dimension $2\left(n_{1}+\cdots+n_{j}-c\right)$. However, $n_{1}, \ldots, n_{\mathrm{ht}\left(N_{\Gamma}\right)}>0$. Therefore, if we define $\pi_{j}^{a}$ (for any $\left.a \in M_{\{\beta\}}\right)$ as in (5.4), then Lemma 5.5.6 implies that for any $j^{\prime} \neq j$, the dimension of the coadjoint orbits associated to the irreducible representations of $N_{\Gamma}^{\beta}$ in the support of $\operatorname{Res}_{N_{\Gamma}}^{P} \pi_{j}^{a}$ is different from the dimension of the coadjoint orbits assoiated to the irreducible representations of $N_{\Gamma}^{\beta}$ in the support of $\operatorname{Res}_{N_{\Gamma}}^{P} \pi_{j^{\prime}}$. This means that

$$
\operatorname{Hom}_{N_{\Gamma}^{\beta}}\left(\operatorname{Res}_{N_{\Gamma}^{\beta}}^{P} \pi_{j}^{a}, \operatorname{Res}_{N_{\Gamma}^{\beta}}^{P} \pi_{j^{\prime}}\right)=\{0\} .
$$

Now we apply Lemma 5.2 .4 with $K=\left[P_{\{\beta\}}, P_{\{\beta\}}\right], K^{\prime}=N_{\Gamma}^{\beta}, \sigma=\pi, \sigma_{1}=\pi_{i}$ and

$$
\sigma_{2}=\bigoplus_{i \neq j} \pi_{i}
$$

It follows that each $\pi_{j}$ is invariant under the action of $M_{\{\beta\}}$. Lemma 5.5.7 is proved.

To finish the proof of Theorem 5.3.2, we note that the parabolic subgroup $P$ in $G$ is maximal, therefore the group generated by $M_{\{\beta\}}$ and $P$ will be equal to $G$. (This follows from the Bruhat-Tits decomposition.) The decomposition of $\pi$ given in Lemma 5.5.5 is preserved by both $P$ and $M_{\{\beta\}}$, and hence by the group generated by them. Therefore the decomposition of Lemma 5.5 .5 is $G$-invariant.

Remark. Let $G$ be as in Theorem 5.3.1, such that the central extension identifying $G$ is as in (1.1). Let $N_{\Gamma}$ be the H-tower subgroup of the group $G$. Let $\pi$ be a unitary 
representation of $G$ of pure rank $k$, where $k \leq \mathrm{ht}\left(N_{\Gamma}\right)$. Consider the subgroup $A_{k}$ of $G$ defined as follows. $A_{k}=p^{-1}\left(\mathbf{A}_{\mathbb{F}}^{\prime}\right)$, where $\mathbf{A}^{\prime}$ is an $\mathbb{F}$-torus inside the maximal split F-torus $\mathbf{A}$ whose Lie algebra is spanned by the coroots $H_{\tilde{\beta}_{1}}, \ldots, H_{\tilde{\beta}_{k}}$. Let $\tau$ be a rankable representation of $N_{\Gamma}$ of rank $k$. The stabilizer $S_{\tau}$ of $\tau$ inside $A_{k}$ is a finite subgroup of $A_{k}$. Moreover, under the action of $A_{k}$ there are only a finite number of orbits of rankable representations of rank $k$. By Mackey theory, the restriction of $\pi$ to $A_{k} \ltimes N_{\Gamma}$ is a direct integral of representations of the form

$$
\operatorname{Ind}_{S_{\tau} \ltimes N_{\Gamma}}^{A_{k} \ltimes N_{\Gamma}} \sigma_{\tau}
$$

where $\sigma_{\tau}$ is irreducible and $\sigma_{\tau \mid N_{\Gamma}}=n_{\tau} \tau$ for some $n_{\tau} \in\{1,2,3, \ldots, \infty\}$. Moreover, by Frobenius reciprocity, $\sigma_{\tau}$ will be a subrepresentation of $\operatorname{Ind}_{N_{\Gamma}}^{S_{\tau} \ltimes N_{\Gamma}} \tau$. Since $S_{\tau}$ is a finite group, there are only a finite number of possibilities for $\sigma_{\tau}$. Since the number of orbits (and hence stabilizers) of rankable representations of rank $k$ under the action of $A_{k}$ is finite, the number of representations of the form (5.10) is finite as well. This implies the following result.

Proposition 5.5.8. Let $\pi$ be a unitary representation of $G$ of pure rank $k$. Then there exists a finite family $\left\{\tau_{1}, \ldots, \tau_{t}\right\}$ of irreducible representations of $A_{k} \ltimes N_{\Gamma}$, independent of $\pi$, such that

$$
\pi_{\mid A_{k} \ltimes N_{\Gamma}}=n_{1} \tau_{1}+\cdots+n_{t} \tau_{t}
$$

where $n_{i} \in\{1,2,3, \ldots, \infty\}$ for each $i$.

\section{Relation With THE OLD THEORY}

6.1. Outline of the old theory. In this section we show how the notion of rank defined in the past sections relates to the existing theory for classical groups. To this end, we show that for the real forms of classical groups, the two notions of rank (the one defined in [Li1] and the one defined in Definition 5.3.3) are equivalent. Here we give a brief outline of the old theory. In classical cases, rank of a representation of the real semisimple group $G$ is defined in terms of its restriction to the centers of nilradicals of maximal parabolic subgroups. One can characterize each of these parabolics with a node in the Dynkin diagram of the restricted root system in a natural way. It turns out that there is a (not necessarily unique) standard parabolic which provides the most refined information about the rank. We will devote this section to exhibiting the coincidence of the two notions of rank on this parabolic. Really the main idea is some slight modification of the fact that the nilradical of the rank parabolic subalgebra contains a maximal isotropic subspace of each of the Heisenberg algebras in the H-tower $N_{\Gamma}$. Our presentation of the results follows the notation of older literature [Hw1], [Li1], Sca].

The notation used in this section is chosen independent of other sections in order to simplify matters and be more coherent with older works. For simplicity we only consider the case $\mathbb{F}=\mathbb{R}$. The general case is essentially the same and will only be more technical. It is more convenient to consider classical groups of different types (in the sense of [Hw5]) separately. Here we quickly review the definition of classical groups of types I and II over a local field, but later we will retain our assumption that $\mathbb{F}=\mathbb{R}$.

Let $\mathbb{F}$ be a local field, $D$ a division algebra over $\mathbb{F}$ with an involution, and $V$ a left vector space over $D$ of dimension $n$. A classical group $G$ is said to be of type II if $G=G L_{D}(V)$. 
From now on, by $(\cdot, \cdot)$ we mean a Hermitian or skew-Hermitian sesquilinear form $(\cdot, \cdot)$ on $V$. A classical group $G$ of type I is the connected component of identity of the stabilizer subgroup of $(\cdot, \cdot)$ inside $G L_{D}(V)$. The real groups of type I which are of our interest here, i.e. those which satisfy the assumptions of Proposition 3.1.1. correspond to the cases where $\mathbb{F}=\mathbb{R}$ and $D=\mathbb{R}, \mathbb{C}$ or $\mathbb{H}$ with their usual involutions.

6.2. Groups of type I. A typical maximal parabolic of these groups can be described as follows. Take a maximal polarization inside $V$, i.e. a maximal set of vectors

$$
\left\{e_{1}, \ldots, e_{r}, e_{1}^{*}, \ldots, e_{r}^{*}\right\}
$$

in $V$ which satisfy

$$
\begin{gathered}
\left(e_{i}, e_{j}\right)=\left(e_{i}^{*}, e_{j}^{*}\right)=0 \\
\left(e_{i}, e_{j}^{*}\right)=\delta_{i, j} .
\end{gathered}
$$

In fact $r$ is equal to the split rank of $G$. For any $k$ let

$$
X_{k}=\operatorname{Span}_{D}\left\{e_{1}, \ldots, e_{k}\right\} \quad, \quad X_{k}^{*}=\operatorname{Span}_{D}\left\{e_{1}^{*}, \ldots, e_{k}^{*}\right\}
$$

and define $V_{k}$ to be $X_{k} \oplus X_{k}^{*}$. Let $P_{k}$ be the subgroup of $G$ that consists of elements which leave the subspace $X_{k}^{*}$ invariant. $P_{k}$ is a parabolic subgroup and the Levi decomposition of $P_{k}$ looks like

$$
P_{k}=G L_{D}\left(X_{k}^{*}\right) G\left(V_{k}^{\perp}\right) N_{k}
$$

where by $G\left(V_{k}^{\perp}\right)$ we mean the stabilizer of $(\cdot, \cdot)$ as a form on $V_{k}^{\perp}$. Here $N_{k}$ is the unipotent radical of $P_{k}$.

$P_{r}$ is the parabolic which provides the most refined information about the rank (in the sense of [Hw1, Li1]).

Definition 6.2.1. Let $r$ be the split rank of $G$. The parabolic $P_{r}$ or its Lie algebra are called the rank parabolic.

The unipotent radical $N_{k}$ is a two-step nilpotent simply connected Lie group and therefore it can be identified with its Lie algebra via the exponential map. From now on, we think of any $N_{k}$ through this identification, and although slightly ambiguous, we use the same notation for its Lie subgroups and their Lie algebras. This is done in order to avoid complicated notation and to keep the presentation as close to the style used in the papers of Howe and Li. We will make it clear whether or not we are using a Lie group or a Lie algerba wherever necessary.

As in [Li1, we have the following exact sequence of Lie algebras:

$$
0 \longrightarrow Z N_{k} \longrightarrow N_{k} \longrightarrow \operatorname{Hom}_{D}\left(V_{k}^{\perp}, X_{k}^{*}\right) \longrightarrow 0
$$

where $Z N_{k}$ is the center of $N_{k} . Z N_{k}$ is isomorphic to

$$
\operatorname{Hom}_{D}^{\text {inv }}\left(X_{k}, X_{k}^{*}\right)
$$

where $\operatorname{Hom}_{D}^{\text {inv }}\left(X_{k}, X_{k}^{*}\right)$ is the $\mathbb{F}$-subspace of elements $T$ of $\operatorname{Hom}_{D}\left(X_{k}, X_{k}^{*}\right)$ satisfying

$$
\forall i, j \in\{1,2, \ldots, k\}, \quad\left(T e_{i}, e_{j}\right)+\left(e_{i}, T e_{j}\right)=0 .
$$


Thus as an $\mathbb{F}$-vector space, the Lie algebra $N_{k}$ can be expressed as

$$
N_{k}=\operatorname{Hom}_{D}\left(V_{k}^{\perp}, X_{k}^{*}\right) \oplus \operatorname{Hom}_{D}^{\text {inv }}\left(X_{k}, X_{k}^{*}\right) .
$$

The isomorphism of $\operatorname{Hom}_{D}\left(V_{k}^{\perp}, X_{k}^{*}\right)$ into the Lie algebra $N_{k}$ can be depicted as

$$
\begin{aligned}
\sim: \operatorname{Hom}_{D}\left(V_{k}^{\perp}, X_{k}^{*}\right) & \mapsto \operatorname{Hom}_{D}(V, V) \\
T & \mapsto \tilde{T}
\end{aligned}
$$

where $\tilde{T}$ is defined as follows:

$$
\begin{array}{ccc}
\tilde{T} v=T v & \text { for } & v \in V_{k}^{\perp} \\
\tilde{T} v=0 & \text { for } & v \in X_{k}^{*} \\
\tilde{T} v=T^{t} v & \text { for } & v \in X_{k} .
\end{array}
$$

Here $T^{t} \in \operatorname{Hom}_{D}\left(X_{k}, V_{k}^{\perp}\right)$ is defined uniquely by

$$
\forall v \in V_{k}^{\perp}, x \in X_{k} \quad(T v, x)+\left(v, T^{t} x\right)=0 .
$$

It turns out that the Heisenberg parabolic $P$ of $G$ is $P_{k_{1}}$, where $k_{1}=2$ for $G=S O_{p, q}$ and $k_{1}=1$ for all other classical cases under consideration. Let $P=L N$ be the Levi decomposition of $P$. Let $M$ be the appropriate simple isotropic factor of $[L, L]$; i.e. we drop the redundant factor of $[L, L]$ which, in (6.1) , corresponds to $G L_{D}\left(X_{k_{1}}^{*}\right)$. Let $\mathfrak{m}$ be the Lie algebra of $M$. For any $k$ define

$$
Y_{k}=\operatorname{Span}\left\{e_{k}, \ldots, e_{r}\right\} \quad, \quad Y_{k}^{*}=\operatorname{Span}\left\{e_{k}^{*}, \ldots, e_{r}^{*}\right\} .
$$

The center of the nilradical of the rank parabolic of $\mathfrak{m}$ is identical to

$$
\operatorname{Hom}_{D}^{\text {inv }}\left(Y_{k_{1}+1}, Y_{k_{1}+1}^{*}\right) \text {. }
$$

The Lie algebra $\operatorname{Hom}_{D}^{\text {inv }}\left(Y_{k_{1}+1}, Y_{k_{1}+1}^{*}\right)$ acts on the Lie algebra $N_{k_{1}}$ through the adjoint action of $\mathfrak{m}$. By Theorem 3.1.1 this action will be trivial on $Z N_{k_{1}}$. The following simple lemma describes this action more explicitly.

Lemma 6.2.2. Let $X \in \operatorname{Hom}_{D}^{\mathrm{inv}}\left(Y_{k+1}, Y_{k+1}^{*}\right)$. Let $Y \in \operatorname{Hom}_{D}\left(V_{k}^{\perp}, X_{k}^{*}\right)$. Then the adjoint action of $\mathfrak{m}$ on $\mathfrak{n}=N_{k}$ is described as

$$
\operatorname{ad}_{X}(\tilde{Y})=-(\tilde{Y X})
$$

where $\tilde{Y X}$ is defined as in (6.3).

Remark. Note that we think of $-Y X$ as an element of $\operatorname{Hom}_{D}\left(V_{k}^{\perp}, X_{k}^{*}\right)$ which is zero on $V_{r}^{\perp}$ and $Y_{k+1}^{*}$.

The restriction of $(\cdot, \cdot)$ to $V_{r}^{\perp}$ is a definite form. Without loss of generality, we may assume that the form is positive definite. Let $\left\{f_{1}, \ldots, f_{n-2 r}\right\}$ be an orthonormal basis for $V_{c}=V_{r}^{\perp}$; consequently $V_{c}=\operatorname{Span}_{D}\left\{f_{1}, \ldots, f_{n-2 r}\right\}$. One can see that $\operatorname{Hom}_{D}\left(V_{k}^{\perp}, X_{k}^{*}\right)$ is equal to

$$
\operatorname{Hom}_{D}\left(Y_{k+1}, X_{k}^{*}\right) \oplus \operatorname{Hom}_{D}\left(Y_{k+1}^{*}, X_{k}^{*}\right) \oplus \operatorname{Hom}_{D}\left(V_{c}, X_{k}^{*}\right) .
$$

We consider the direct sum decomposition (6.4) inside $N_{k}$ (see (6.2) ) We observe that: 
Lemma 6.2.3. In the direct sum decomposition 6.4, the third summand commutes with the first two summands, and the adjoint action of $\operatorname{Hom}_{D}^{\mathrm{inv}}\left(Y_{k+1}, Y_{k+1}^{*}\right)$ on the third summand is trivial.

Now take $k=k_{1}$. Then the direct sum

$$
\operatorname{Hom}_{D}\left(Y_{k_{1}+1}, X_{k_{1}}^{*}\right) \oplus \operatorname{Hom}_{D}\left(Y_{k_{1}+1}^{*}, X_{k_{1}}^{*}\right) \oplus \operatorname{Hom}_{D}^{\text {inv }}\left(X_{k_{1}}, X_{k_{1}}^{*}\right)
$$

is a Lie subalgebra of $N_{k_{1}}$, and also a Heisenberg algebra with a polarization given by the first two summands in (6.5). We denote the Lie algebra in (6.5) (and also its corresponding Lie group) by $\bar{N}_{k_{1}}$. The Lie bracket when restricted to the polarization is described as follows.

Lemma 6.2.4. Let $X \in \operatorname{Hom}_{D}\left(Y_{k_{1}+1}, X_{k_{1}}^{*}\right)$ and $Y \in \operatorname{Hom}_{D}\left(Y_{k_{1}+1}^{*}, X_{k_{1}}^{*}\right)$. Then $[\tilde{X}, \tilde{Y}]$ is an element of $\operatorname{Hom}_{D}^{\mathrm{inv}}\left(X_{k_{1}}, X_{k_{1}}^{*}\right)$ given by

$$
[\tilde{X}, \tilde{Y}]=X Y^{t}-Y X^{t}
$$

where $X^{t} \in \operatorname{Hom}_{D}\left(X_{k_{1}}, Y_{k_{1}+1}^{*}\right)$ and $Y^{t} \in \operatorname{Hom}_{D}\left(X_{k_{1}}, Y_{k_{1}+1}\right)$ are uniquely determined as follows

$$
\begin{aligned}
& \forall i \leq k_{1} \text { and } \forall j>k_{1},\left(X^{t} e_{i}, e_{j}\right)+\left(e_{i}, X e_{j}\right)=0 \\
& \forall i \leq k_{1} \text { and } \forall j>k_{1},\left(Y^{t} e_{i}, e_{j}^{*}\right)+\left(e_{i}, Y e_{j}^{*}\right)=0 .
\end{aligned}
$$

Proof. Follows immediately from $[\tilde{X}, \tilde{Y}]=\tilde{X} \tilde{Y}-\tilde{Y} \tilde{X}$ where $\tilde{X}$ and $\tilde{Y}$ are defined as in (6.3).

The adjoint action of $\operatorname{Hom}_{D}^{\text {inv }}\left(Y_{k_{1}+1}, Y_{k_{1}+1}^{*}\right)$ on $\bar{N}_{k_{1}}$ is given by Lemma 6.2.2, This action normalizes $\bar{N}_{k_{1}}$ and takes $\operatorname{Hom}_{D}\left(Y_{k_{1}+1}^{*}, X_{k_{1}}^{*}\right)$ to $\operatorname{Hom}_{D}\left(Y_{k_{1}+1}, X_{k_{1}}^{*}\right)$.

At this point we come back to nilpotent groups and their representations. Consider an irreducible representation $\rho_{1}$ of the Heisenberg group $N_{k_{1}}$ with (nontrivial) central character $\chi_{1}$. From the orthogonal decomposition obtained in (6.5) it follows that the restriction of $\rho_{1}$ to the group $\bar{N}_{k_{1}}$ decomposes into a direct integral of representations of this latter Heisenberg group with the same central character. We study the restriction of a rankable representation of rank one of the H-tower unipotent radical of $G$ to its subgroup

$$
\bar{N}_{k_{1}} \rtimes \operatorname{Hom}_{D}^{\text {inv }}\left(Y_{k_{1}+1}, Y_{k_{1}+1}^{*}\right) .
$$

This restriction is a direct integral of representations of the latter group obtained by extending the irreducible representation of $\bar{N}_{k_{1}}$ with central character $\chi_{1}$ to $\bar{N}_{k_{1}} \rtimes$ $\operatorname{Hom}_{D}^{\text {inv }}\left(Y_{k_{1}+1}, Y_{k_{1}+1}^{*}\right)$ as suggested by Proposition 2.3.1. This is because Lemma 6.2 .3 implies that as subspaces of $N_{k_{1}}$,

$$
\left[\operatorname{Hom}_{D}^{\mathrm{inv}}\left(Y_{k_{1}+1}, Y_{k_{1}+1}^{*}\right), \operatorname{Hom}_{D}\left(V_{c}, X_{k_{1}}^{*}\right)\right]=\{0\}
$$

and the Weil representation is functorial. (See (1.15) of [Hw1] for a precise meaning of functoriality.)

Since $\operatorname{Hom}_{D}^{\text {inv }}\left(Y_{1}, Y_{1}^{*}\right)$ is an abelian Lie group, any unitary representation of this group can be described as a direct integral of unitary characters. $\operatorname{Hom}_{D}^{\mathrm{inv}}\left(Y_{1}, Y_{1}^{*}\right)$ is isomorphic 
to some $\mathbb{R}^{p}$; so its group of unitary characters can be naturally identified to $\operatorname{Hom}_{D}^{\mathrm{inv}}\left(Y_{1}^{*}, Y_{1}\right)$ via the $\mathbb{F}$-bilinear form

$$
\beta(A, B)=\operatorname{tr}(A B) .
$$

Definition 6.2.5. The rank of a character of $\operatorname{Hom}_{D}^{\mathrm{inv}}\left(Y_{1}, Y_{1}^{*}\right)$ is the $\mathbb{F}$-rank of the element in $\operatorname{Hom}_{D}^{\text {inv }}\left(Y_{1}^{*}, Y_{1}\right)$ which corresponds to it via the bilinear form in (6.6).

Definition 6.2.6. (See [Hw1, [Li1]) Let $\pi$ be a unitary representation of $G$. $\pi$ is said to have rank $k$ if and only if $\pi_{\mid \operatorname{Hom}_{D}^{\mathrm{inv}}\left(Y_{1}, Y_{1}^{*}\right)}$ decomposes into a direct integral of characters of rank equal to $k$.

The following proposition is a key result of this section.

Proposition 6.2.7. The restriction of a rankable representation of rank one to

$$
\operatorname{Hom}_{D}^{\text {inv }}\left(Y_{1}, Y_{1}^{*}\right)
$$

is supported on characters whose rank is equal to $k_{1}$.

Proof. The polarization for the group $\bar{N}_{k_{1}}$ has the structure of a $D$-vector space. Therefore, similar to (2.5), we can realize the representation $\rho_{1}$ of $\bar{N}_{k_{1}}$ on

$$
L^{2}\left(\operatorname{Hom}_{D}\left(Y_{k_{1}+1}^{*}, X_{k_{1}}^{*}\right)\right)
$$

and then extend it to $\operatorname{Hom}_{D}^{\text {inv }}\left(Y_{k_{1}+1}, Y_{k_{1}+1}^{*}\right)$. In Lemma 6.2.8 below, we denote elements of the Lie algebras by $X, Y, \ldots$ and the corresponding elements in the Lie groups by $e^{X}, e^{Y}, \ldots$

Lemma 6.2.8. The action of the extension of $\rho_{1}$ is described as below.

(a) For any $X \in \operatorname{Hom}_{D}\left(Y_{k_{1}+1}, X_{k_{1}}^{*}\right), Y \in \operatorname{Hom}_{D}\left(Y_{k_{1}+1}^{*}, X_{k_{1}}^{*}\right)$

$$
\left(\rho_{1}\left(e^{X}\right) f\right)(Y)=\chi_{1}\left(e^{[Y, X]}\right) f(Y)
$$

(b) For any $X \in \operatorname{Hom}_{D}^{\text {inv }}\left(Y_{k_{1}+1}, Y_{k_{1}+1}^{*}\right), Y \in \operatorname{Hom}_{D}\left(Y_{k_{1}+1}^{*}, X_{k_{1}}^{*}\right)$

$$
\left(\rho_{1}\left(e^{X}\right) f\right)(Y)=\chi_{1}\left(e^{\frac{1}{2}[Y, Y X]}\right) f(Y)
$$

(c) For any $X \in \operatorname{Hom}_{D}^{\text {inv }}\left(X_{k_{1}}, X_{k_{1}}^{*}\right), Y \in \operatorname{Hom}_{D}\left(Y_{k_{1}+1}^{*}, X_{k_{1}}^{*}\right)$

$$
\left(\rho_{1}\left(e^{X}\right) f\right)(Y)=\chi_{1}\left(e^{X}\right) f(Y) .
$$

Proof. This is an almost immediate consequence of the Schrödinger model for the realization of Weil representation. See [Hw6].

Let $X \in \operatorname{Hom}_{D}^{\text {inv }}\left(Y_{k_{1}+1}, Y_{k_{1}+1}^{*}\right)$ and $Y \in \operatorname{Hom}_{D}\left(Y_{k_{1}+1}^{*}, X_{k_{1}}^{*}\right)$. For all $i \leq k_{1}$ and $j>k_{1}$,

$$
\begin{gathered}
\left(e_{j},(Y X)^{t} e_{i}\right)=-\left(Y X e_{j}, e_{i}\right)=\left(X e_{j}, Y^{t} e_{i}\right) \\
=-\left(e_{j}, X Y^{t} e_{i}\right)=\left(e_{j},-X Y^{t} e_{i}\right)
\end{gathered}
$$

which implies that $(Y X)^{t}=-X Y^{t}$. Thus the equation in part (b) of Lemma 6.2.8 can be simplified as

$$
\left(\rho_{1}(X) f\right)(Y)=\chi_{1}\left(e^{-Y X Y^{t}}\right) f(Y)
$$

We would like to have a single formula instead of parts (a), (b) and (c) of Lemma 6.2.8. To this end, we define the linear operator $S=S(Y)$ such that

$$
S: Y_{1}^{*} \mapsto X_{k_{1}}^{*}
$$


by

$$
\begin{gathered}
S e_{i}^{*}=e_{i}^{*} \quad \text { if } \quad i \leq k_{1} \\
S e_{i}^{*}=Y e_{i}^{*} \quad \text { if } \quad i>k_{1} .
\end{gathered}
$$

We have the following lemma.

Lemma 6.2.9. Let $X \in \operatorname{Hom}_{D}^{\text {inv }}\left(Y_{1}, Y_{1}^{*}\right)$. Let $f$ be a function such that

$$
f \in L^{2}\left(\operatorname{Hom}_{D}\left(Y_{k_{1}+1}^{*}, X_{k_{1}}^{*}\right)\right) .
$$

Then

$$
\rho_{1}\left(e^{X}\right) f(Y)=\chi_{1}\left(e^{-S X S^{t}}\right) f(Y)
$$

where $S^{t}$ is defined as in Lemma 6.2.4.

Proof. Applying $\left(e_{i}, S e_{j}^{*}\right)+\left(S^{t} e_{i}, e_{j}^{*}\right)=0$, one can see that for any $i \leq k_{1}$

$$
S^{t} e_{i}=-e_{i}+Y^{t} e_{i}
$$

and thus for any $X \in \operatorname{Hom}_{D}\left(Y_{k_{1}+1}, X_{k_{1}}^{*}\right)$

$$
\begin{array}{r}
-S X S^{t} e_{i}=-S \tilde{X}\left(-e_{i}+Y^{t} e_{i}\right)= \\
(-S)\left(-X^{t}\right) e_{i}-S X Y^{t} e_{i}= \\
Y X^{t} e_{i}-X Y^{t} e_{i}=[Y, X] e_{i}
\end{array}
$$

which proves $-S X S^{t}=[Y, X]$.

For any $X \in \operatorname{Hom}_{D}^{\text {inv }}\left(Y_{k_{1}+1}, Y_{k_{1}+1}^{*}\right)$ we have

$$
-S X S^{t} e_{i}=-S \tilde{X}\left(-e_{i}+Y^{t} e_{i}\right)=-S X Y^{t} e_{i}=-Y X Y^{t} e_{i}
$$

which proves $-S X S^{t}=-Y X Y^{t}$.

Finally, when $X \in \operatorname{Hom}_{D}^{\text {inv }}\left(X_{k_{1}}, X_{k_{1}}^{*}\right)$

$$
-S X S^{t} e_{i}=-S X\left(-e_{i}+Y^{t} e_{i}\right)=S X e_{i}=X e_{i}
$$

which proves $-S X S^{t}=X$. This completes the proof.

To complete the proof of Proposition 6.2.7 note that via the identification described in (6.6), the character $\chi\left(e^{X}\right)=\chi_{1}\left(e^{-S^{t} X S}\right)$ corresponds to an element

$$
-\frac{a}{k_{1}} S^{t} V S \quad a \in i \mathbb{R}-\{0\}
$$

where

is defined as

$$
V: X_{k_{1}}^{*} \mapsto X_{k_{1}}
$$

$$
V e_{l}^{*}=(-1)^{l+1} e_{k_{1}-l+1}^{*} \quad \text { for any } \quad 1 \leq l \leq k_{1} .
$$

It is easy to see that (6.7) is an element of $\operatorname{Hom}_{D}^{\text {inv }}\left(Y_{1}^{*}, Y_{1}\right)$ of rank $k_{1}$, even when its domain is restricted to $X_{k_{1}}$.

The following theorem shows that in groups of type I, the two notions of rank are essentially the same.

Theorem 6.2.10. Let $\pi$ be an irreducible representation of a classical group $G$ of type I. Let $N_{\Gamma}$ be the H-tower subgroup of $G$ (see (3.4)). 
- Assume that the rank of $\pi$ in the sense of Definition 6.2.6 is less than $\operatorname{ht}\left(N_{\Gamma}\right) \times k_{1}$. Then $\pi$ has rank $k$ in the sense of Definition 5.3 .3 if and only if $\pi$ has rank $k k_{1}$ in the sense of Definition 6.2.6.

- If the rank of $\pi$ in the sense of Definition 5.3.3 is equal to ht $\left(N_{\Gamma}\right)$, then rank of $\pi$ in the sense of Definition 6.2.6 is $\mathrm{ht}\left(N_{\Gamma}\right) \times k_{1}$ or higher.

Proof. Since $\pi$ is supported on rankable representations, the only thing we have to show is that a rankable representation of rank $k$ (in the sense of Definition 4.1.1), when restricted to $\operatorname{Hom}_{D}^{\text {inv }}\left(Y_{1}^{*}, Y_{1}\right)$, decomposes as a direct integral of characters of rank $k k_{1}$. By Proposition 6.2.7 this is true when $k=1$.

Next consider a rankable representation $\rho$ of rank $k>1$, say

$$
\rho=\rho_{1} \otimes \cdots \otimes \rho_{k}
$$

Elementary properties of tensor product imply that the restriction of $\rho$ to $\operatorname{Hom}_{D}^{\text {inv }}\left(Y_{1}, Y_{1}^{*}\right)$ is a direct integral of characters of the form

$$
\phi_{1} \cdot \phi_{2}
$$

where the characters $\phi_{1}$ and $\phi_{2}$ are constituents of the direct integral decomposition of $\rho_{1}$ and $\rho_{2} \otimes \cdots \otimes \rho_{k}$, when restricted to $\operatorname{Hom}_{D}^{\text {inv }}\left(Y_{1}, Y_{1}^{*}\right)$, respectively. But if $\phi_{i}(i \in\{1,2\})$ corresponds to $A_{i} \in \operatorname{Hom}_{D}^{\mathrm{inv}}\left(Y_{1}^{*}, Y_{1}\right)$ via (6.6) , then $\phi_{1} \cdot \phi_{2}$ corresponds to $A_{1}+A_{2}$. Since $\rho_{2} \otimes \cdots \otimes \rho_{k}$ and therefore any possible $\phi_{2}$ is a trivial representation when restricted to $N_{k_{1}}$, any possible $A_{2}$ is really an element of $\operatorname{Hom}_{D}^{\text {inv }}\left(Y_{k_{1}+1}^{*}, Y_{k_{1}+1}\right)$ which is extended trivially on $X_{k_{1}}$ to $Y_{1}^{*}$. However, at the end of the proof of Proposition 6.2.7 it was shown that any such character $\phi_{1}$ corresponds to some element of $\operatorname{Hom}_{D}^{\text {inv }}\left(Y_{1}^{*}, Y_{1}\right)$ which is of rank $k_{1}$ even when its domain is restricted to $X_{k_{1}}^{*}$. It is now easy to show that we have

$$
\operatorname{rank}\left(A_{1}+A_{2}\right)=\operatorname{rank}\left(A_{1}\right)+\operatorname{rank}\left(A_{2}\right)=k_{1}+\operatorname{rank}\left(A_{2}\right) .
$$

An induction on $k$ completes the proof.

Example. Let $G=S O(6,6)$. Let $\pi$ be an irreducible unitary representation of $G$. Then the rank of $\pi$ in the sense of Definition 6.2.6 can be $0,2,4$ or 6 . The rank of $\pi$ in the sense of Definition 5.3.3 can be 0,1 or 2. The following chart shows how the ranks correspond to each other:

\begin{tabular}{r|l} 
Definition 6.2.6 & Definition 5.3.3 \\
\hline 0 & 0 \\
2 & 1 \\
4 & 2 \\
6 & 2
\end{tabular}

Now let $G=S O(5,11)$. Then we have a similar chart for the rank of $\pi$.

\begin{tabular}{r|l} 
Definition 6.2.6 & Definition 5.3 .3 \\
\hline 0 & 0 \\
2 & 1 \\
4 & 2
\end{tabular}

Therefore the correspondence of ranks may or may not be one to one. It is an easy exercise to determine in which cases the correspondence is actually one to one. 
6.3. $\mathbf{S L}_{\mathbf{l + 1}}(\mathbb{R})$. Let $G=S L_{l+1}(\mathbb{R})$, the group of linear transformations on the $l+1$ dimensional vector space $V$ with a fixed basis

$$
\left\{e_{1}, \ldots, e_{l+1}\right\} \text {. }
$$

Set $r=\left\lfloor\frac{l+1}{2}\right\rfloor$. For any $k$ let $P_{k}$ be the maximal parabolic of $G$ which is represented by matrices of the form

$$
\left[\begin{array}{cc}
A & B \\
0 & C
\end{array}\right]
$$

where

$$
A \in G L_{k}(\mathbb{R}) \quad, \quad C \in G L_{l+1-k}(\mathbb{R}) \quad, \quad \text { and } B \in \mathrm{M}_{k \times(l+1-k)}(\mathbb{R}) .
$$

Therefore

$$
P_{k}=S\left(G L_{k}(\mathbb{R}) \times G L_{l+1-k}(\mathbb{R})\right) \cdot N_{k}
$$

where

$$
N_{k}=\operatorname{Hom}\left(\operatorname{Span}\left(\left\{e_{k+1}, \ldots, e_{l+1}\right\}\right), \operatorname{Span}\left(\left\{e_{1}, \ldots, e_{k}\right\}\right)\right) .
$$

The parabolic of $G$ which gives the most refined rank is $P_{r}$, and henceforth we focus our attention to $P_{r}$. Let

$$
X_{k}=\operatorname{Span}\left(\left\{e_{2}, \ldots, e_{k}\right\}\right) \quad, \quad Y_{k}=\operatorname{Span}\left(\left\{e_{k}, \ldots, e_{l}\right\}\right) .
$$

The Heisenberg parabolic subgroup of $G$ is $P_{1} \cap P_{l}$, and a polarization of the Lie algebra of its unipotent radical $N$ is a direct sum of

$$
\operatorname{Hom}\left(X_{r}, \mathbb{R} e_{1}\right) \oplus \operatorname{Hom}\left(\mathbb{R} e_{l+1}, Y_{r+1}\right)
$$

and

$$
\operatorname{Hom}\left(Y_{r+1}, \mathbb{R} e_{1}\right) \oplus \operatorname{Hom}\left(\mathbb{R} e_{l+1}, X_{r}\right) .
$$

The second summand lies inside the nilradical of the Lie algebra of $P_{r}$. Its center is isomorphic to

$$
\operatorname{Hom}\left(\mathbb{R} e_{l+1}, \mathbb{R} e_{1}\right) \text {. }
$$

As before we are interested in description of the restriction of a representation $\rho_{1}$ of $N$ to

$$
\operatorname{Hom}\left(\operatorname{Span}\left(\left\{e_{r+1}, \ldots, e_{l+1}\right\}\right), \operatorname{Span}\left(\left\{e_{1}, \ldots, e_{r}\right\}\right)\right) \text {. }
$$

We identify the dual of

$$
\operatorname{Hom}\left(\operatorname{Span}\left(\left\{e_{r+1}, \ldots, e_{l+1}\right\}\right), \operatorname{Span}\left(\left\{e_{1}, \ldots, e_{r}\right\}\right)\right)
$$

with itself via the bilinear form

$$
\beta(X, Y)=\operatorname{tr}\left(X^{t} Y\right) .
$$

The rank of a unitary character of

$$
\operatorname{Hom}\left(\operatorname{Span}\left(\left\{e_{r+1}, \ldots, e_{l+1}\right\}\right), \operatorname{Span}\left(\left\{e_{1}, \ldots, e_{r}\right\}\right)\right)
$$

is defined to be the rank of the linear transformation which corresponds to it via the bilinear form $\beta$.

We write any

$$
Y \in \operatorname{Hom}\left(X_{r}, \mathbb{R} e_{1}\right) \oplus \operatorname{Hom}\left(\mathbb{R e}_{l+1}, Y_{r+1}\right)
$$

naturally as $Y=Y_{1} \oplus Y_{2}$. Define

$$
Y_{1}^{+} \in \operatorname{Hom}\left(\operatorname{Span}\left(\left\{e_{1}, \ldots, e_{r}\right\}\right), \mathbb{R} e_{1}\right)
$$


by

$$
\begin{aligned}
& Y_{1}^{+} e_{1}=e_{1} \\
& Y_{1}^{+} e_{j}=Y_{1} e_{j} \quad \text { for any } j>1 .
\end{aligned}
$$

Similarly,

$$
Y_{2}^{+} \in \operatorname{Hom}\left(\mathbb{R} e_{l+1}, \operatorname{Span}\left(\left\{e_{r+1}, \ldots, e_{l+1}\right\}\right)\right)
$$

is defined to be

$$
Y_{2}^{+} e_{l+1}=-Y_{2} e_{l+1}+e_{l+1} .
$$

We can prove the following version of Lemma 6.2.9.

Lemma 6.3.1. Let $\rho_{1}$ be a representation of $N$ with central character $\chi_{1}$ realized on

$$
\mathcal{H}=L^{2}\left(\operatorname{Hom}\left(X_{r}, \mathbb{R} e_{1}\right) \oplus \operatorname{Hom}\left(\mathbb{R} e_{l+1}, Y_{r+1}\right)\right)
$$

as in section 2.1.

For any

$$
X \in \operatorname{Hom}\left(\operatorname{Span}\left(\left\{e_{r+1}, \ldots, e_{l+1}\right\}\right), \operatorname{Span}\left(\left\{e_{1}, \ldots, e_{r}\right\}\right)\right),
$$

we have

$$
\rho_{1}(X) f(Y)=\chi_{1}\left(e^{Y_{1}^{+} X Y_{2}^{+}}\right) f(Y) .
$$

Proof. We will write $Y$ as $Y_{1} \oplus Y_{2}$ according to the polarization given in the statement of the lemma. Based on the Schrödinger model, if $X \in \operatorname{Hom}\left(Y_{r+1}, X_{r}\right)$, then the action of $X$ on the function $f$ at a point $Y$ is multiplication by the character

$$
\chi_{1}\left(e^{-\frac{1}{2}[Y, Y X]}\right)=\chi_{1}\left(e^{-Y_{1} X Y_{2}}\right) .
$$

If $X=X_{1} \oplus X_{2} \in \operatorname{Hom}\left(Y_{r+1}, \mathbb{R} e_{1}\right) \oplus \operatorname{Hom}\left(\mathbb{R} e_{l+1}, X_{r}\right)$ then $[X, Y]=-X_{1} Y_{2}+Y_{1} X_{2}$ and the action of $X$ is by the character

$$
\chi_{1}\left(e^{-X_{1} Y_{2}+Y_{1} X_{2}}\right)
$$

and if $X$ belongs to the center of $N$ then clearly the action will be by the character

$$
\chi_{1}\left(e^{X}\right) .
$$

The statement of the lemma follows by a simple calculation.

One can see that by the duality provided via bilinear form $\beta$, The restriction of a representation of rank one to the nilradical of $P_{r}$ is a direct integral of characters which correspond to linear operators of the form $Y_{2}^{+} Y_{1}^{+}$, which have rank one (in the usual sense) even when the domain is restricted to $\mathbb{R} e_{l+1}$. Proof of the following theorem (which shows the equivalence of the two notions of rank) is similar to that of Theorem 6.2 .10 .

Theorem 6.3.2. Let $G=S L_{l+1}(\mathbb{R})$. Then the restriction of a pure-rank representation of $G$ of rank $k$ (in the sense of Definition 5.3.3) to the abelian nilradical of $P_{r}$ is supported on unitary characters of rank $k$.

Remark. Note that when $l$ is even, the maximum rank of the unitary characters is $\frac{l}{2}$, which is the same as the height of the H-tower subgoup of $G$. However, for odd $l$, the maximum rank of unitary characters exceeds the height of the H-tower group by one. 


\section{REFERENCES}

[AV] Adams, J. and Vogan, D. (Editors) Representation theory of Lie groups, Lectures from the Graduate Summer School held in Park City, UT, July 13-31, 1998. IAS/Park City Mathematics Series, $8,2000$.

[Ad] Adams, J. Non-linear covers of real groups, preprint, 2004.

[Bor] Borel, A., Linear algebraic groups, Springer-Verlag, New York, 1991.

[Bou] Bourbaki, N., Lie groups and Lie algebras. Chapters 4-6. Translated from the 1968 French original by Andrew Pressley. Elements of Mathematics (Berlin). Springer-Verlag, Berlin, 2002.

[Br] Brown, I. D., Dual topology of a nilpotent Lie group, Ann. Sci. de l'E.N.S. $4^{e}$ serie, no. 6, (1973), pp 407-411.

[CG] Corwin, L. J. and Greenleaf, F. P., Representations of nilpotent Lie groups and their applications, Cambridge university press, 1990.

[De] Deodhar, Vinay V. On central extensions of rational points of algebraic groups, Amer. J. Math. 100 (1978), no. 2, 303-386.

[Du] Duflo, M., Théorie de Mackey pour les groupes de Lie algébrique, Acta Math. 149 (1982), 152-213.

[DS] Dvorsky, A and Sahi, S., Tensor products of singular representations and an extension of the $\theta$-correspondence. Selecta Math. (N.S.) 4 (1998), no. 1, 11-29.

[GS] Gan, W. T. and Savin, Gordan On minimal representations, Representation Theory 9 (2005), 46-93.

[GRS] Ginzburg, David and Rallis, Stephen and Soudry, David, A tower of theta correspondences for $G_{2}$. Duke Math. J. 88 (1997), no. 3, 537-624.

[GW] Gross, B. H. and Wallach, N. R., On quaternionic discrete series representations, and their continuations, J. Reine Angew. Math. 481 (1996), 73-123.

[Hel] Helgason, S. Differential geometry, Lie groups, and symmetric spaces, AMS, 2000.

[Hw1] Howe, R., On a notion of rank for unitary representations of the classical groups, Harmonic analysis and group representations, 223-331, Liguori, Naples, 1982.

[Hw2] Howe, R. On the role of the Heisenberg group in harmonic analysis., Bull. Amer. Math. Soc. (N.S.) 3 (1980), no. 2, 821-843.

[Hw3] Howe, R. Small unitary representations of classical groups, Group representations, ergodic theory, operator algebras, and mathematical physics (Berkeley, Calif., 1984), 121-150, Math. Sci. Res. Inst. Publ., 6, Springer, New York, 1987.

[Hw4] Howe, R., Automorphic forms of low rank, Noncommutative harmonic analysis and Lie groups (Marseille, 1980), pp. 211-248, Lecture Notes in Math., 880, Springer, Berlin-New York, 1981.

[Hw5] Howe, R., $\theta$-series and invariant theory, Automorphic forms, representations and $L$-functions (Proc. Sympos. Pure Math., Oregon State Univ., Corvallis, Ore., 1977), Part 1, pp. 275-285

[Hw6] Howe, R., Oscillator representation, analytic preliminaries, unpublished notes, 1977.

[HM] Howe, R. and Moore, C. Asymptotic properties of unitary representations, J. Funct. Anal. 32 (1979), no. 1, 72-96.

[HRW] Howe, R., Ratcliff, G. and Wildberger, N., Symbol mappings for certain nilpotent groups, Lie group representations, III (College Park, Md., 1982/1983), 288-320, Lecture Notes in Math., 1077, Springer, Berlin, 1984.

[Hu] Humphreys, J. E., Linear algebraic groups, Graduate Texts in Mathematics 21, Springer-Verlag, New York-Heidelberg, 1975.

[Jo] Joseph, A. A preparation theorem for the prime spectrum of a semisimple Lie algebra, J. Algebra 48 (1977), no. 2, 241-289.

[Kaz] Kazhdan, David, Some applications of the Weil representation. J. Analyse Mat. 32 (1977), 235248.

[Kr1] Kirillov, A. A., Unitary representations of nilpotent Lie groups (Russian), Uspehi Mat. Nauk 17 1962 no. 4 (106), 57-110.

[Kr2] Kirillov, A. A., Lectures on the orbit method, Graduate Studies in Mathematics 64, American Mathematical Society, Providence, RI 2004.

[Ki] Kirillov, A. A. Jr., Merits and demerits of the orbit method, (English. English summary) Bull. Amer. Math. Soc. (N.S.) 36 (1999), no. 4, 433-488. 
[Li1] Li, J.-S., Singular unitary representations of classical groups, Invent. Math. 97 (1989), no. 2, $237-255$.

[Li2] Li, J. -S., On the classification of irreducible low rank unitary representations of classical groups, Compositio Math. 71 (1989), no. 1, 29-48.

[Li3] Li, J. -S., Automorphic forms with degenerate Fourier coefficients, Amer. J. Math. 119 (1997), no. $3,523-578$.

[LZ] Li, J. S., Zhu, C. B., On the decay of matrix coefficients for exceptional groups, Math. Ann. 305, no. 2, 249-270 (1996).

[LS] Loke, H. Y. and Savin, G., Rank and matrix coefficents for simply-laced groups, preprint, 2005.

[Mac1] Mackey, G. W., The theory of unitary group representations, University of Chicago Press, 1976.

[Mac2] Mackey, G. W., Induced representations of locally compact groups I, Ann. of Math. (2) 55, (1952). $101-139$.

[MS] Magaard, K. and Savin, G., Exceptional $\Theta$-correspondences. I. Compositio Math. 107 (1997), no. $1,89-123$.

[Mo1] Moore, Calvin C., Decomposition of unitary representations defined by discrete subgroups of nilpotent groups, Ann. of Math. (2) $821965,146-182$.

[Mo2] Moore, Calvin C., Extensions and low dimensional cohomology theory of locally compact groups I, II. Trans. Amer. Math. Soc. 1131964 40-63.

[Oh] Oh, H., Uniform pointwise bounds for matrix coefficients of unitary representations and applications to Kazhdan constants, Duke Math. J. 113 no. 1, 133-192 (2002).

[PR] Platonov, V. and Rapinchuk, A., Algebraic groups and number theory. Translated from the 1991 Russian original by Rachel Rowen. Pure and Applied Mathematics, 139. Academic Press, Inc., Boston, MA, 1994.

[Sa] Salmasian, H., Isolatedness of minimal representations and minimal decay of matrix coefficients, submitted.

[Sca] Scaramuzzi, R., A notion of rank for unitary representations of general linear groups, Trans. Amer. Math. Soc. 319 (1990), no. 1, 349-379.

[Se] Serre, J. P., Lie algebras and Lie groups, Lecture Notes in Mathematics 1500, Springer, Berlin, 1992.

[Tay] Taylor, M. E., Noncommutative harmonic analysis, AMS Mathematical Surveys and Monographs, no. 22, 1986.

[Tor] Torasso, P. Méthode des orbites de Kirillov-Duflo et représentations minimales des groupes simples sur un corps local de caractéristique nulle (French), Duke Math. J. 90 (1997), no. 2, 261-377.

[Ti] Tits, Jacques Tabellen zu den einfachen Lie Gruppen und ihren Darstellungen (German), Lecture Notes in Mathematics 40, Springer-Verlag, Berlin-New York 1967.

[We1] Weil, A., Basic number theory. Third edition. Die Grundlehren der Mathematischen Wissenschaften, Band 144. Springer-Verlag, New York-Berlin, 1974.

[We2] Weil, A. Sur certains groupes d'opérateurs unitaires, Acta Math. 111 (1964), 143-211.

[Ws] Weissman, M.H., The Fourier-Jacobi map and small representations, Represent. Theory 7 (2003), $275-299$.

Queen's University, Department of Mathematics and Statistics, Jeffery Hall, University Avenue, Kingston, Ontario K7L 3N6, Canada

E-mail address: hadi@mast.queensu.ca 